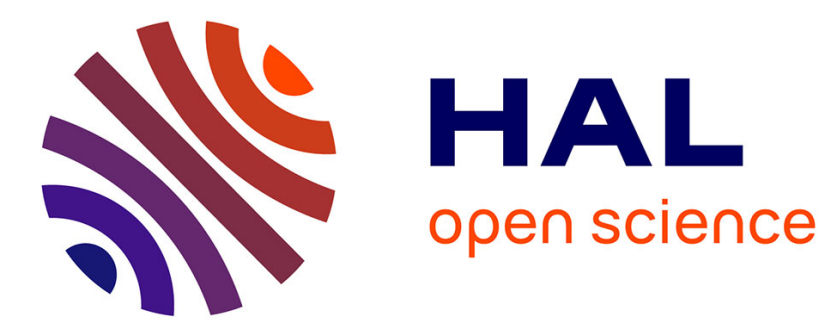

\title{
Un atelier de potier médiéval au coeur de la ville fortifiée de Thérouanne
}

Olivier Blamangin, Vaiana Vincent

\section{To cite this version:}

Olivier Blamangin, Vaiana Vincent. Un atelier de potier médiéval au coeur de la ville fortifiée de Thérouanne. Revue du Nord, 2011, 393 (5), pp.261-276. 10.3917/rdn.393.0261 . hal-02139168

\section{HAL Id: hal-02139168 https://hal-inrap.archives-ouvertes.fr/hal-02139168}

Submitted on 3 Jun 2019

HAL is a multi-disciplinary open access archive for the deposit and dissemination of scientific research documents, whether they are published or not. The documents may come from teaching and research institutions in France or abroad, or from public or private research centers.
L'archive ouverte pluridisciplinaire HAL, est destinée au dépôt et à la diffusion de documents scientifiques de niveau recherche, publiés ou non, émanant des établissements d'enseignement et de recherche français ou étrangers, des laboratoires publics ou privés. 


\section{UN ATELIER DE POTIER MÉDIÉVAL AU CEUR DE LA VILLE FORTIFIÉE DE THÉROUANNE}

Olivier Blamangin et Vaiana Vincent

Association Revue du Nord | « Revue du Nord »

2011/5 N³93 | pages 261 à 276

ISSN 0035-2624

Article disponible en ligne à l'adresse :

https://www.cairn.info/revue-du-nord-2011-5-page-261.htm

Distribution électronique Cairn.info pour Association Revue du Nord.

(C) Association Revue du Nord. Tous droits réservés pour tous pays.

La reproduction ou représentation de cet article, notamment par photocopie, n'est autorisée que dans les limites des conditions générales d'utilisation du site ou, le cas échéant, des conditions générales de la licence souscrite par votre établissement. Toute autre reproduction ou représentation, en tout ou partie, sous quelque forme et de quelque manière que ce soit, est interdite sauf accord préalable et écrit de l'éditeur, en dehors des cas prévus par la législation en vigueur en France. Il est précisé que son stockage dans une base de données est également interdit. 


\section{Un atelier de potier médiéval au cœur de la ville fortifiée de Thérouanne}

Un four de potier, daté de la fin du XIV eu du début $\mathrm{du} \mathrm{XV}^{\mathrm{e}}$ siècle, a été partiellement fouillé à l'occasion d'un diagnostic réalisé en juin et juillet 2006 à Thérouanne, rue Saint-Jean ${ }^{1}$. C'est la première fois qu'une activité de production céramique est ainsi attestée intra muros. En dépit du caractère limité de l'intervention archéologique, qui n'a pas donné lieu à la prescription d'une fouille plus exhaustive, l'état de conservation de la structure de cuisson et la qualité de l'ensemble céramique issu de son comblement justifient la publication de ces données. Elles permettent d'esquisser un premier profil des productions locales au Bas Moyen Âge.

\section{CONTEXTE DE LA DÉCOUVERTE}

Thérouanne fut successivement chef-lieu de la Civitas Morinorum (Cité des Morins), puis siège épiscopal jusqu'à la destruction de la ville sur ordre de Charles Quint en $1553^{2}$. Depuis le début des années quatre-vingt-dix, les opérations d'archéologie préventive se sont multipliées sur le territoire de la commune, qui nous permettent aujourd'hui de mieux appréhender la topographie de la ville aux différentes périodes de l'Antiquité ${ }^{3}$ et du Moyen Âge. Pour autant, nos connaissances restent très fragmentaires pour un site de cette importance.

L'intervention archéologique, réalisée par l'Inrap en bordure de la rue Saint-Jean (parcelle cadastrale AB 239), est située au cœur de la ville fortifiée médiévale, sur le versant exposé au sud de la vallée de la Lys (fig. 1). La parcelle est à $350 \mathrm{~m}$ environ au sud-est de la cathédrale et à moins de $100 \mathrm{~m}$ de l'ancienne place du marché, dont l'emprise probable est fossilisée dans le cadastre de la ville.

\footnotetext{
*. - Olivier Blamangin, Inrap Nord-Picardie, Halma-Ipel 8164 (CNRS, Lille 3, MCC); Vaiana VINCENT, Inrap Nord-Picardie. 1. - BLAMANGIN 2011.
}

Conformément aux prescriptions du Service régional de l'archéologie, le diagnostic a consisté en la réalisation de «fenêtres » de décapage mécanique à l'emplacement de trois pavillons qui devaient être construits (sondages 1 à 3 , fig. 2), en respectant la cote de fond de forme $(-0,70 \mathrm{~m})$. Un sondage ponctuel (sondage 4) plus profond a également été réalisé pour une reconnaissance de la stratigraphie. La totalité de la surface décapée est couverte d'un épais remblai moderne (briques, tuiles, gravats, etc.) qui apparaît immédiatement sous la terre végétale, résultat du nivellement du secteur après destruction de la ville au milieu du $\mathrm{XVI}^{\mathrm{e}}$ siècle. Les structures archéologiques mises au jour apparaissent immédiatement sous ce niveau de remblai, d'épaisseur variable $(0,20$ à $0,30 \mathrm{~m}$ au niveau du sondage 1 , supérieur à $0,50 \mathrm{~m}$ au niveau du sondage 3). Les différences dans les cotes d'apparition des vestiges témoignent d'une topographie médiévale un peu plus marquée et plus irrégulière que la topographie actuelle.

Aucune structure archéologique n'a été mise au jour dans le sondage 3, l'épaisseur de la terre végétale et du remblai moderne dépassant la cote de fond de forme. Un grand bâtiment du Bas Moyen Âge et ses annexes (fig. 3) ont été partiellement dégagés au niveau du sondage 1 . D'une largeur comprise entre 6,50 et $7 \mathrm{~m}$ et d'une longueur supérieure à $11 \mathrm{~m}$, l'ensemble est orienté SO-NO, perpendiculairement à l'axe de la rue Saint-Jean. Il se prolonge au-delà des limites de décapage. Les bases de murs sont construites en briques jaunes et rouges, associées à des blocs calcaires grossièrement taillés, des rognons de silex et, parfois, de petits blocs de grès ou des tuiles plates, liés par un mortier jaune (M1 à M6). Les soubassements les moins arasés (M1, M2, M4) sont

\footnotetext{
2. - Voir notamment Bernard 1980; Delmaire 1976, 1984, 1994 ; RYDER, TOCK 2010.

3. - Blamangin et al. 2012.
} 


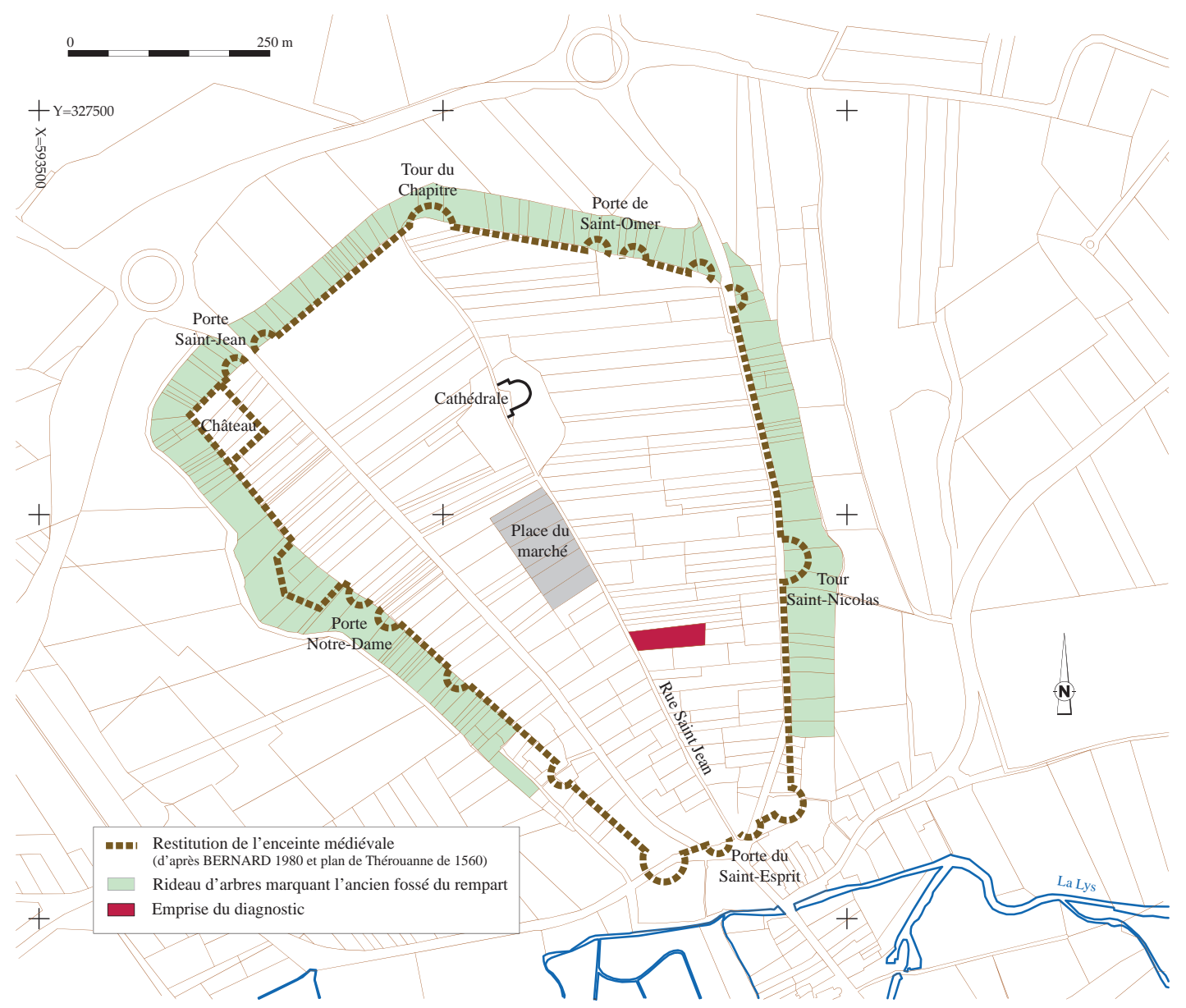

FIG. 1. - Vieille ville de Thérouanne et localisation du diagnostic archéologique. Infographie O. Blamangin.

conservés sur une hauteur supérieure à $0,3 \mathrm{~m}$. Ils étaient destinés à recevoir une élévation à pans de bois, comme en témoignent les nombreux fragments de torchis rubéfiés issus des niveaux de destruction. Des reprises de maçonneries montrent des réfections successives. Deux espaces distincts sont clairement identifiables. Le premier, délimité par les murs M1, $\mathrm{M} 2$, M5 et M6, forme une pièce de $33 \mathrm{~m}^{2}$, avec un seuil d'entrée (dalle de grès) au NE. Le second espace, probablement une cour intérieure, est d'une surface supérieure à $25 \mathrm{~m}^{2}$. Il est bordé, au sud et au nord, par deux appentis. Des couches de tuiles et d'argile rubéfiée, bien circonscrites à la grande pièce et à l'appentis sud-ouest, scellent les niveaux de sol et témoignent d'une destruction violente par le feu de tout ou d'une partie du bâti. Au sud-est de ce bâtiment principal, deux murs perpendiculaires, reprenant les mêmes orientations, ont été partiellement dégagés. Réalisés à sec en gros blocs calcaires, silex et tuiles, ils pourraient appartenir à un second bâtiment contemporain ou antérieur à l'édifice principal.
La céramique issue des niveaux de destruction et du nettoyage des sols du bâtiment principal appartient à un horizon de la fin du $\mathrm{Xv}^{\mathrm{e}}$ ou du début du $\mathrm{XVI}^{\mathrm{e}}$ siècle, mais le mobilier ne permet pas de caractériser la nature de l'occupation. Nous sommes probablement en présence d'un habitat civil correspondant au dernier état de la ville médiévale avant sa destruction au milieu du XVI ${ }^{\mathrm{e}}$ siècle.

\section{LE FOUR DE POTIER MÉDIÉVAL}

Un four de potier médiéval, antérieur à cet habitat civil, a été mis au jour au niveau du sondage 2 . Il est situé à une vingtaine de mètres de la rue Saint-Jean. Sa localisation sur une parcelle en front de rue peut sans doute s'expliquer par les nécessités d'approvisionnement en matière première (combustible, argile, etc.) de l'atelier.

Le four est installé perpendiculairement à la rue dans une fosse oblongue. Seule la partie excavée de la structure de cuisson est conservée. L'aire de chauffe 

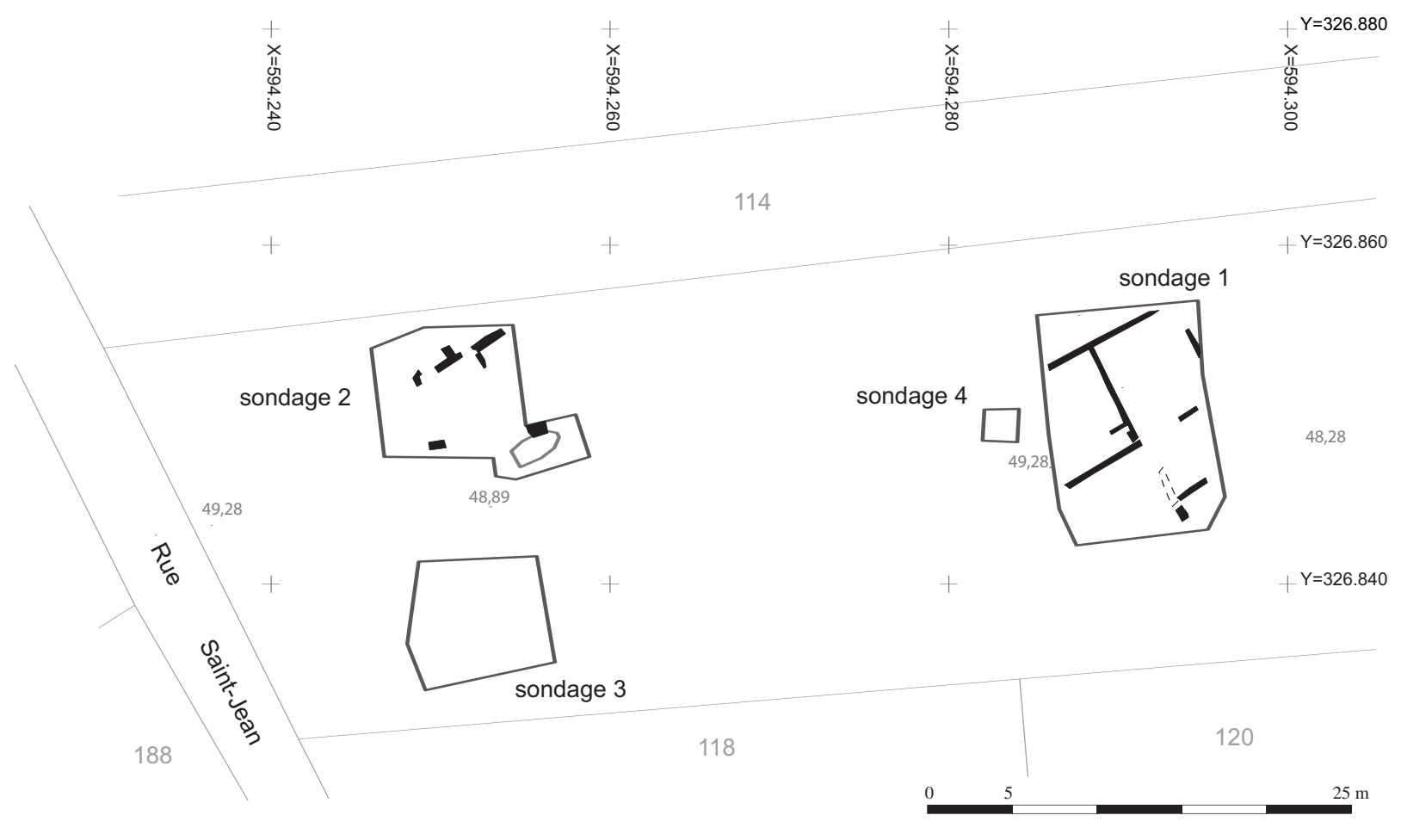

FIG. 2. - Plan général - localisation des sondages. Relevé topographique : R. Debiak, DAO : D. Favier.

n'a pu être dégagée et se développe vers le nord-est, au-delà des limites d'emprise du décapage. La chambre de chauffe, «en obus » $(3 \times 1,40 \mathrm{~m})$, est conservée sur une profondeur maximale de $0,70 \mathrm{~m}$ (fig. 4 et 5). Ses parois verticales sont indurées sur 3 à $5 \mathrm{~cm}$ d'épaisseur, de couleur gris bleuté. Le limon jaune de l'encaissant présente une auréole de chauffe bien marquée sur 0,10 à $0,25 \mathrm{~m}$ de large. Elle s'ouvre au nord-est sur un alandier unique dont la voûte est conservée (fig. 6). Elle est partiellement recoupée par la fondation en brique d'un mur appartenant vraisemblablement à un édifice du dernier état de la ville médiévale, non dégagé en plan.

Deux murets $(2,50 \mathrm{~m} \times 0,20 \mathrm{~m})$ partagent la chambre de chauffe en trois conduits d'inégale largeur. Ces murets étaient destinés à recevoir des boudins d' argile cuite disposés horizontalement pour supporter la charge, comme l'atteste l'un d'eux, conservé en place (fig. 7). Une cinquantaine de fragments de ces boudins ont été recueillis à l'occasion de la fouille du comblement de la chambre de chauffe. De section circulaire (6 à $8 \mathrm{~cm}$ de diamètre), ils présentent le plus souvent des traces de surcuisson (éclatement, fissures, aspect grésé). Le seul boudin complet mesure $0,33 \mathrm{~m}$ de longueur. Les fragments sont légèrement incurvés

FIG. 3. - Plan général du sondage 1. DAO : D. Favier.

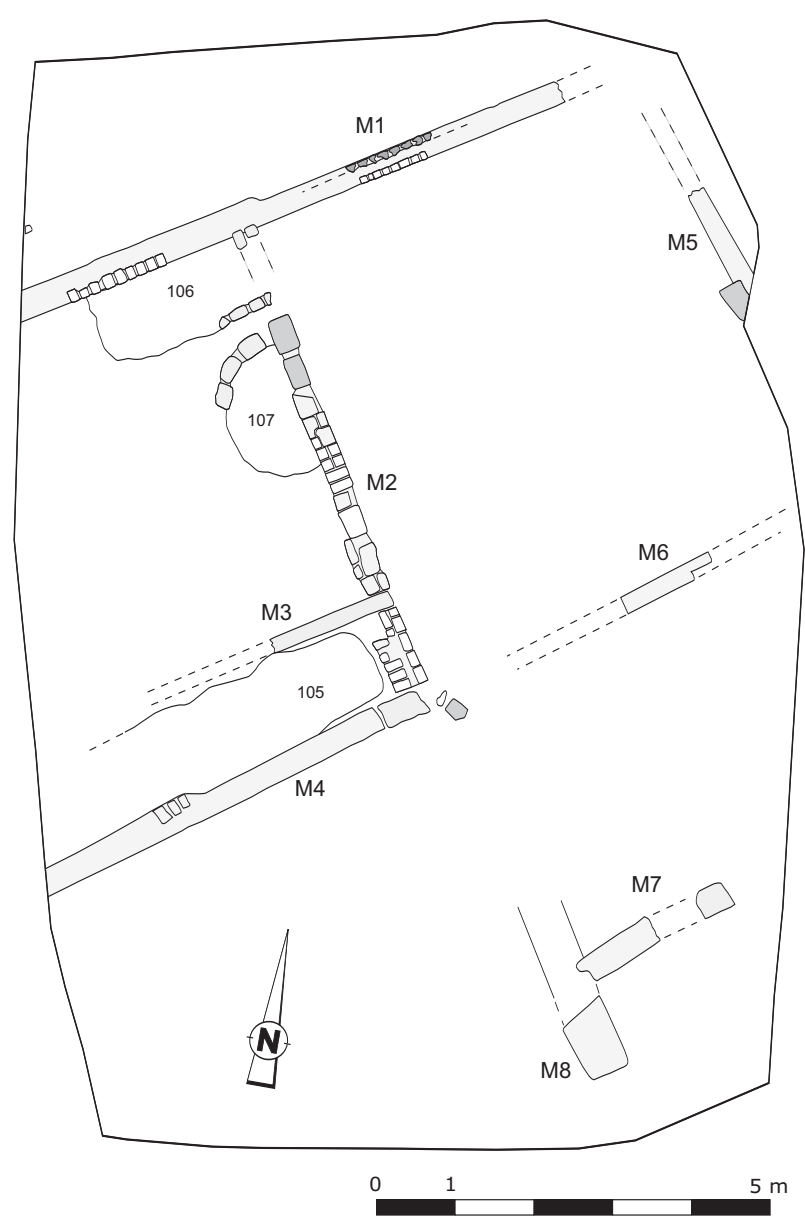




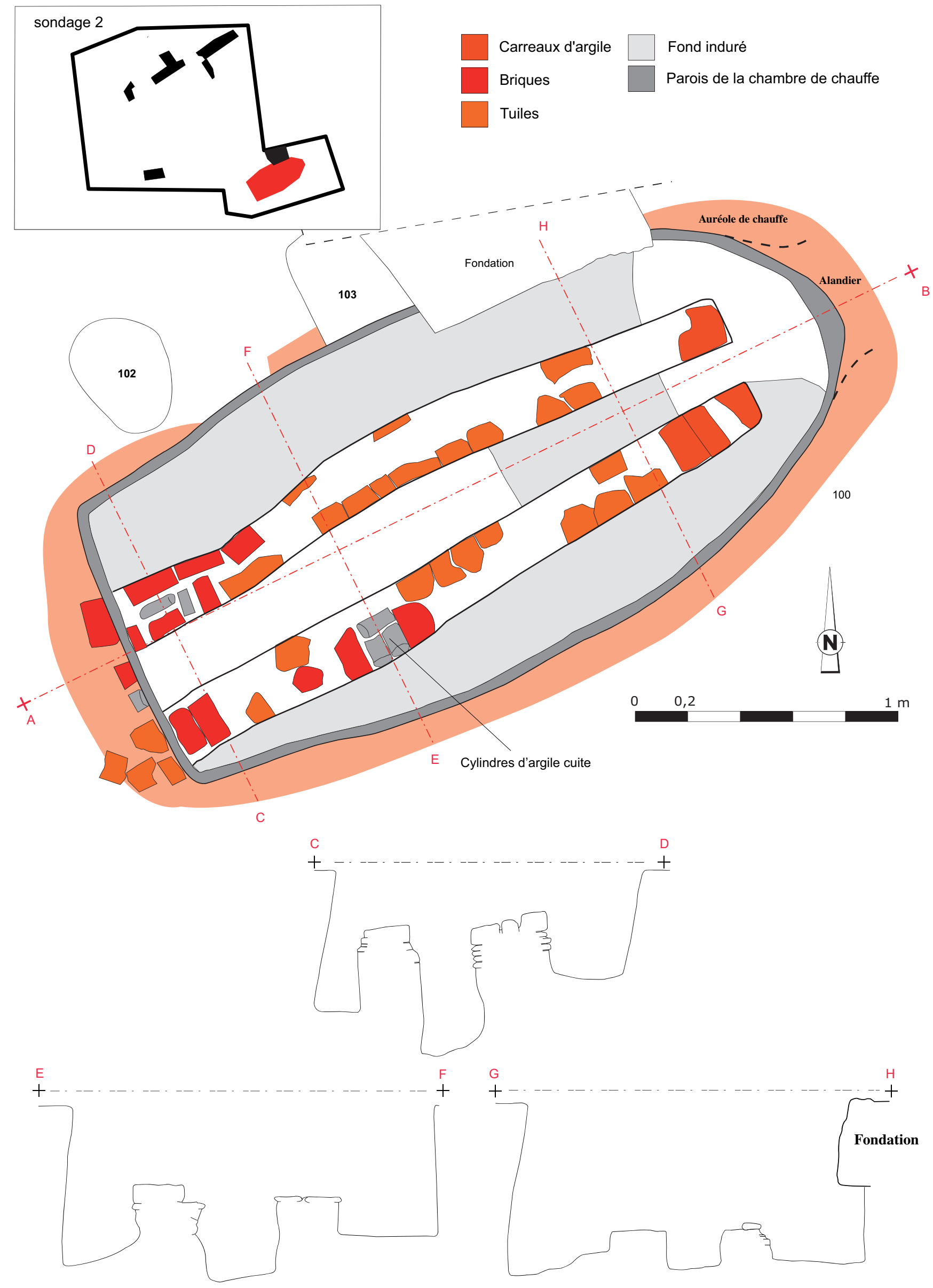

FIG. 4. - Plan et profils de la chambre de chauffe du four. DAO : O. Blamangin et D. Favier. 
et toutes les extrémités sont biseautées, ce qui permet d'enchâsser « en force » les boudins entre les murets et les parois de la chambre de chauffe. Aucune trace d'arrachement, qui pourrait indiquer un scellement à l'argile des barres de support de la charge, n'a été observée.

Le sol induré est partiellement arasé au niveau du canal de chauffe central et à l'entrée de la chambre de chauffe par les opérations de curage de la structure de cuisson. Le fond des conduits de chauffe latéraux présente un pendage assez marqué vers la gueule du four, en particulier pour le conduit nord: 0,44 à 0,56 m de profondeur sur l'arrière de la chambre de chauffe, $0,70 \mathrm{~m}$ vers l'avant. Le fond du conduit de chauffe central, plus profond, est plan, mais cette différence de niveau peut, pour partie, résulter des opérations de curage évoquées ci-dessus.

Les murets partiellement arasés (traces d'arrachement) semblent montés en escalier. Leur construction est soignée, réalisée principalement en carreaux de terre cuite, en tuiles et en briques, noyés dans l'argile. On notera l'utilisation ponctuelle de petits blocs calcaire et de sections de boudins de terre cuite en réemploi (fig. 8). La hauteur conservée de ces murets par rapport au fond du canal central ne dépasse pas 0,14 m à l'extrémité nord-est, vers la gueule du four, contre $0,50 \mathrm{~m}$ à l'arrière de la chambre de chauffe. Le muret nord a visiblement subi une déformation mécanique. Le plan « en obus » de la chambre, l'inclinaison du sol et ces murets en escalier permettent sans doute une diffusion homogène de la chaleur.

Sur l'arrière de la chambre de chauffe, l'auréole de rubéfaction est plus large. Des tuiles, des briques et des sections de cylindres en terre cuite sont disposés en plan; elles pourraient servir de base à un aménagement spécifique de l'élévation du four (cheminée ?) à son extrémité la plus éloignée du foyer (fig. 9).

Le comblement du four intervient rapidement après son abandon (fig. 10). Le remplissage inférieur (US 109) est essentiellement composé de fragments de parois rubéfiées issues de la destruction de la voûte du four. Ces fragments de parois sont beaucoup moins nombreux dans le comblement supérieur (US 101) de la structure de cuisson. Une fine couche de charbons et de cendres est conservée sur le fond de la structure, au niveau de l'alandier.

\section{LES PRODUCTIONS}

Seul le mobilier céramique provenant du comblement du four de potier est présenté dans le cadre de cet article. Si quelques éléments sont probablement en situation primaire (fig. 7), l'essentiel du mobilier est

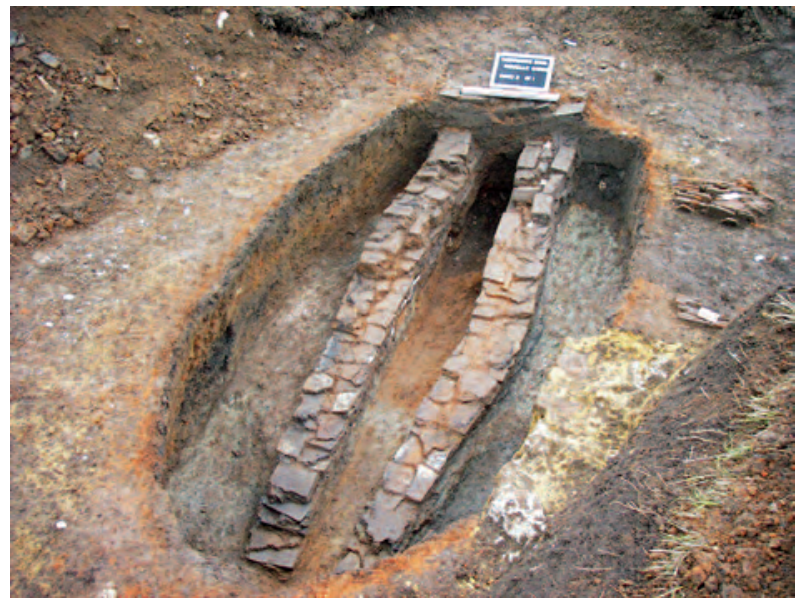

FIG. 5. - Vue de la chambre de chauffe du four depuis le NE. Cliché : O. Blamangin.

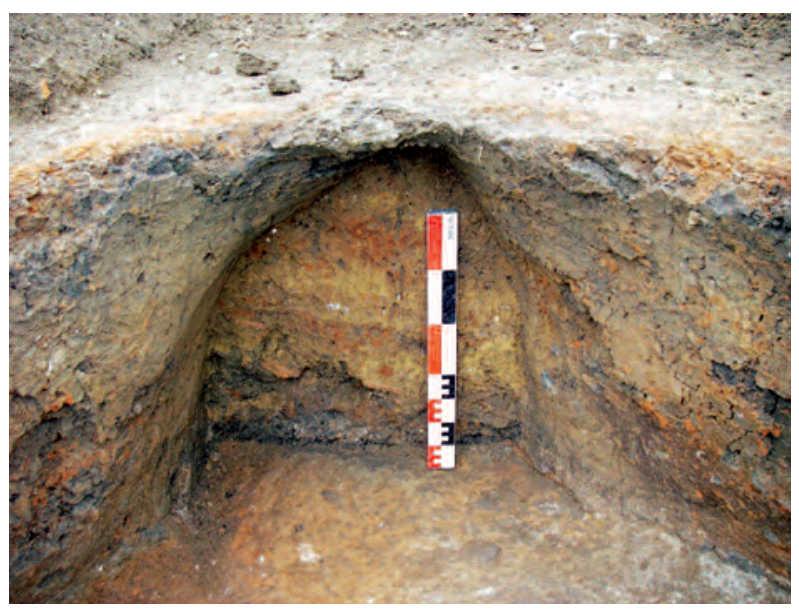

FIG. 6. - Alandier du four. Cliché : O. Blamangin.

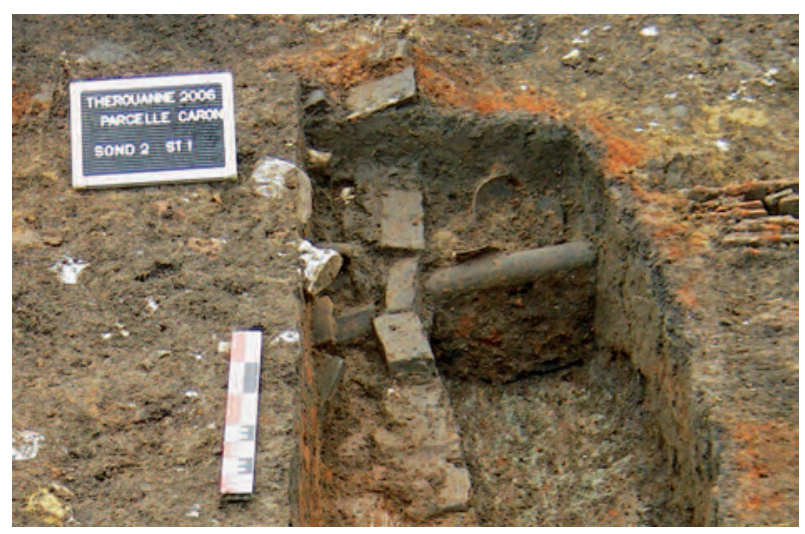

FIG. 7. - Boudin d'argile destiné à supporter la charge enchasser en force entre le muret et la paroi de la chambre de chauffre. Cliché : O. Blamangin. 


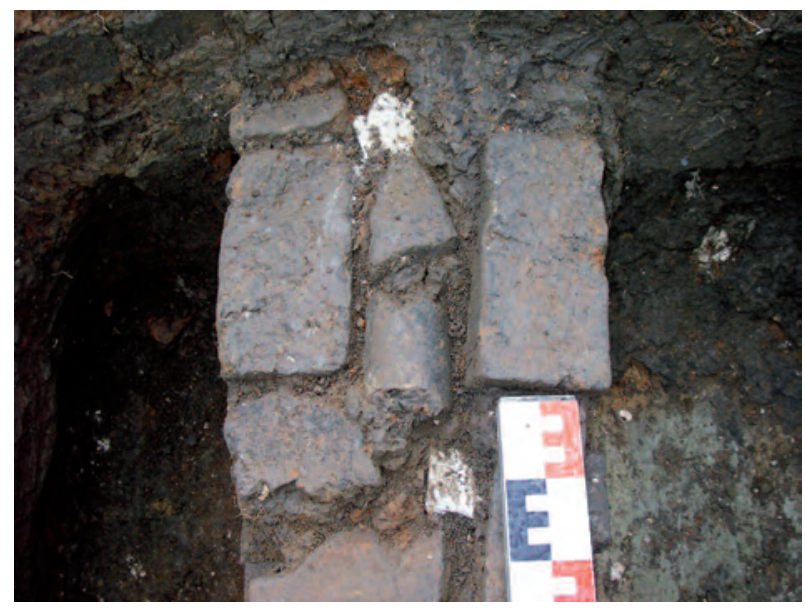

FIG. 8. - Sections de boudin d'argile en réemploi dans le muret nord de la chambre de chauffe.

Cliché : O. Blamangin.

en dépôt secondaire et correspond à la phase d'abandon du four. La présence, dans le même remplissage, d'un grand nombre d'éléments d'architecture du four (boudins d'argile cuite, fragments de parois) indiquent un comblement rapide. Ce contexte, l'importance du lot (590 tessons pour 103 individus, soit $70 \%$ du mobilier total prélevé à l'occasion du diagnostic), sa grande homogénéité (récurrence des types et des pâtes) et des éléments de "contamination » extérieure peu nombreux (importations de longue ou moyenne distance) sont autant d'indices d'une production locale, dont on s'attachera à établir un premier profil. On notera cependant l'absence de rebuts de cuisson évidents (éclatement, déformation ou « coup de feu »), quelques individus présentant des traces d'utilisation (suie) et la présence de restes de

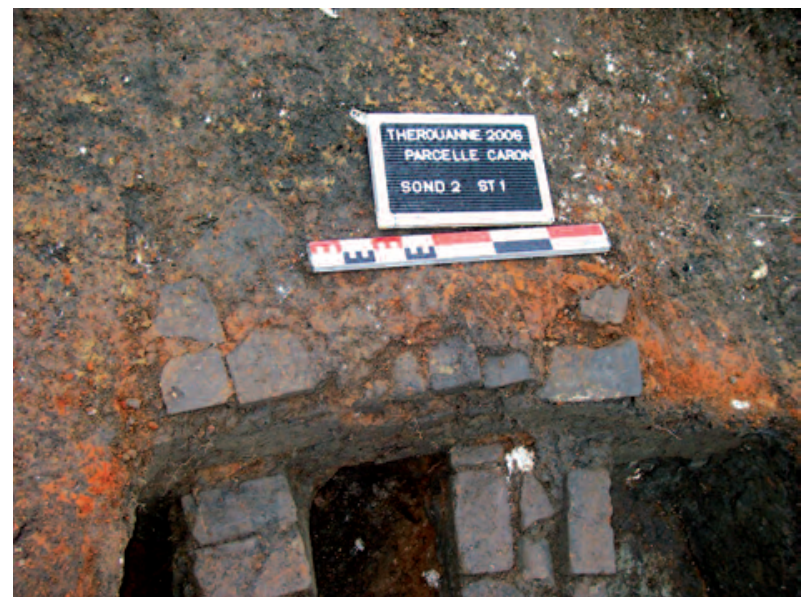

FIG. 9. - Base de l'aménagement de l'élévation du four à son extrémité la plus éloignée du foyer.

Cliché : O. Blamangin.

faune dans le comblement qui nous permettent de conclure à une réutilisation de la chambre de chauffe du four en dépotoir domestique. Ce lot de céramique doit donc être interprété comme d'origine mixte, associant rejets de production et de consommation. Le mobilier, bien qu'issu des deux phases de comblement de la chambre de chauffe (US 101 et 109), présente une parfaite homogénéité, ce qui nous conduit à proposer une étude d'ensemble.

Pour cette étude, les comptages ont été réalisés en individualisant les lèvres $(\mathrm{L})$, panses $(\mathrm{P})$, fonds $(\mathrm{F})$, éléments de préhension (EP) et les éléments verseurs (EV) par catégorie. La base du comptage repose sur le nombre de restes (NR) et sur le nombre minimum d'individus (NMI). Ce dernier a été déterminé uniquement

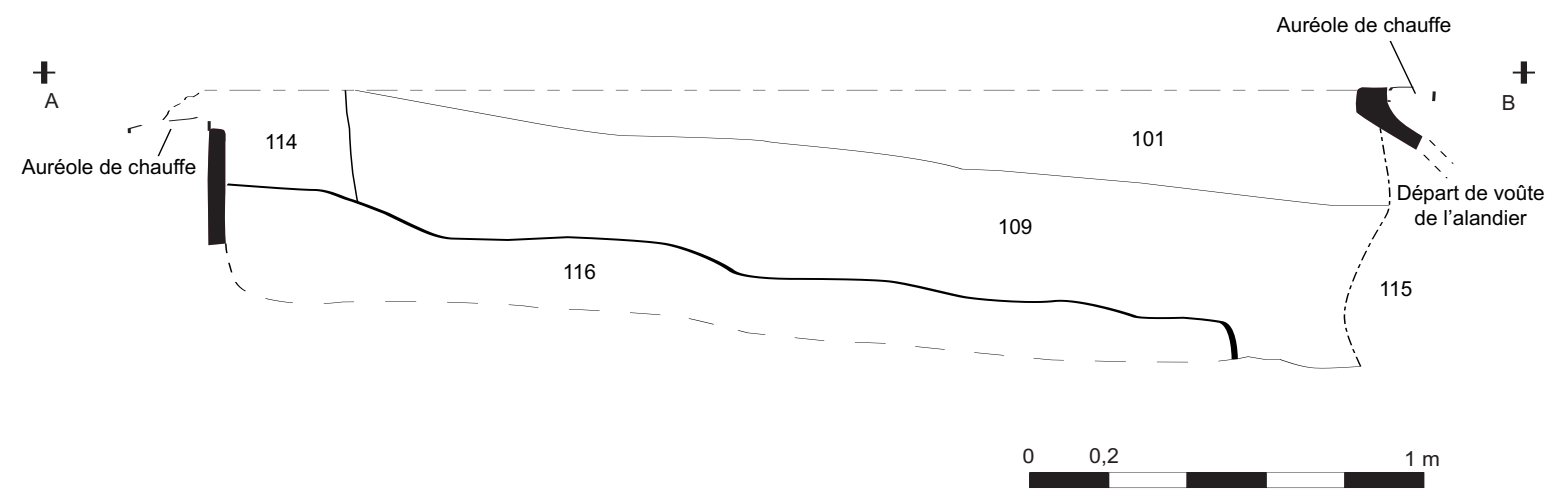

FIG. 10. - Coupe de la chambre de chauffe du four. DAO : D. Favier.

101. Limon sableux gris, framents d'argile rubéfiée, charbon de bois, fragments de tuiles, nodules de mortier jaune, petits blocs de craie ; 109. Argile rubéfiée, nodules de craie brûlée, charbons de bois, boulettes de limon jaune ; 114. Limon gris, charbons de bois, fragments argile rubéfiée, boulettes de limon jaune ; 115 . Limon jaune à beige, charbons de bois, nodules de terres cuites ; 116. Muret latéral (briques, tomettes en terre cuite, blocs de craie liés par de l'argile. 


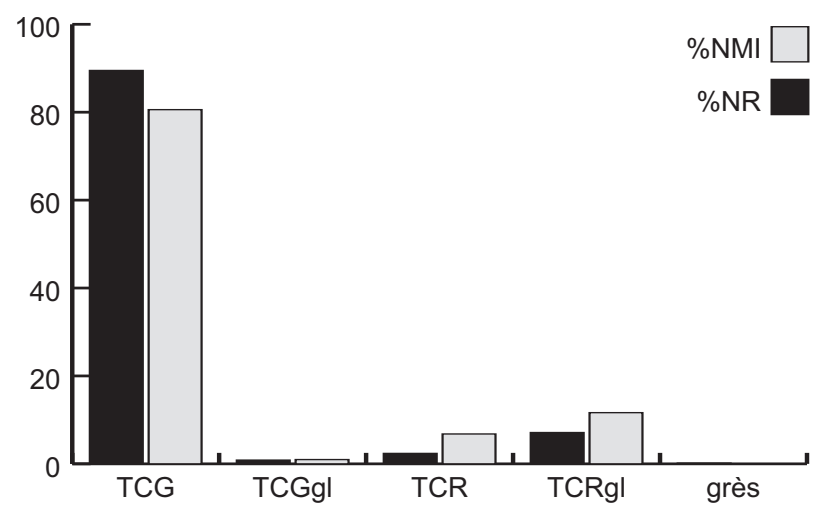

FIG. 11. - Répartition en pourcentage des NR et NMI selon les différentes catégories du mobilier issu du comblement de la chambre de chauffe.

à partir des lèvres, après recollage de celles-ci ${ }^{4}$. Un numéro d'inventaire est attribué à chaque individu qui fait l'objet d'une caractérisation de pâte, sur cassure fraîche, à la loupe binoculaire. Chacune des « fabriques » identifiées est décrite à partir des observations macroscopiques de la surface et du cœur du tesson (granulométrie, nature des inclusions, couleur, fréquence, morphologie, dureté $)^{5}$.

\subsection{Caractérisation technique}

Le mobilier céramique provenant du comblement du four se répartit en cinq catégories qui se distinguent par le mode de cuisson et par les différents types de décor (fig. 11, tab. 1). En mode réducteur, la terre cuite grise sans glaçure (TCG) représente 80,6\% du corpus en NMI. La céramique est bien cuite, sonnante, à pâte fine, sans inclusion visible à l'œil nu, avec une couleur grise en surface et à cœur bien marquée. La présence de glaçure dans ce mode réducteur (TCGgl) est anecdotique, avec seulement un individu : une tèle à bandeau peu saillant, de type T1, à glaçure verte coulée. S'agit-il d'une adaptation technique liée à cette fonction ou simplement d'un raté de cuisson ? Quoiqu'il en soit, les vingt-deux autres individus présentant ce même type de lèvre sont tous en TCG. Le mode oxydant représente $18,4 \%$ de l'ensemble et se répartit entre $6,8 \%$ de terre cuite rouge (TCR) et $11,7 \%$ d'éléments présentant une glaçure (TCRgl). Tout comme la TCG, la céramique est bien cuite, avec une couleur rouge franche. La glaçure est majoritairement coulée. Un seul individu, un couvercle de type C1 (fig. 15), dispose d'une glaçure projetée. La cou-

4. - ArCelin, Tuffreau-Libre 1998, p. VIII.

5. - Pour ce faire, nous nous sommes appuyés sur la fiche de description de la « collection de référence des fabriques gallo-romaines » du

\begin{tabular}{lrrrr}
\hline & NR & \% NR & NMI & \% NMI \\
\hline TCG & 528 & $89,5 \%$ & 83 & $80,6 \%$ \\
\hline TCGgl & 5 & $0,8 \%$ & 1 & $1,0 \%$ \\
\hline TCR & 14 & $2,4 \%$ & 7 & $6,8 \%$ \\
\hline TCRgl & 42 & $7,1 \%$ & 12 & $11,7 \%$ \\
\hline grès & 1 & $0,2 \%$ & & \\
\hline Total & $\mathbf{5 9 0}$ & $100 \%$ & $\mathbf{1 0 3}$ & $100 \%$ \\
\hline
\end{tabular}

Tableau 1. - Données quantitatives de la figure 11.

leur des glaçures se partage de manière assez égale entre le vert et l'orange et peut parfois emprunter des teintes plus brunes. On notera également la présence d'un fragment de grès d'importation (origine Siegburg?), issu du comblement supérieur de la chambre de chauffe (US 101).

\subsection{Groupes de pâtes}

Une caractérisation des pâtes a pu être effectuée à la loupe binoculaire avec un grossissement x16 puis x35. Quatre groupes ont ainsi pu être mis en évidence (fig. 12) pour le mobilier céramique et les éléments d'architecture (boudins de support de la charge, carreaux et briques) issus du comblement du four. Les deux premiers (THER1 et THER2) permettent probablement de caractériser la production céramique locale. Les pâtes de type THER3 appartiennent vraisemblablement à une "fabrique » d'importation. THER4 se rencontre exclusivement sur des éléments d'architecture, sans doute également de production locale.

Le groupe THER1 caractérise des céramiques cuites en mode oxydant comme réducteur. Il s'agit, de très loin, du groupe de pâtes le plus largement représenté avec $93 \%$ du corpus. On le retrouve également, sensiblement dans les mêmes proportions, dans l'horizon plus tardif de l'habitat (fin du $\mathrm{Xv}^{\mathrm{e}}$ et le début du $\mathrm{XVI}^{\mathrm{e}}$ siècle). Il se caractérise, en mode réducteur comme en mode oxydant, par une couleur homogène, du cœur aux bords extérieur et intérieur. Son toucher est lisse. La structure d'ensemble est assez lâche et présente de nombreuses inclusions de quartz subarrondis à anguleux, de couleur blanche, parfois transparents, de petite ou de moyenne taille. On observe également des nodules noirs très brillants, anguleux, assez petits et en petite quantité. Enfin, elle se caractérise

Centre de recherche d'archéologie nationale (CRAN) de Louvain-laNeuve (Belgique). Cf. MÉTHOdes D'ANALYSE DE LA TERRE CUITE 1999, p. 33. 


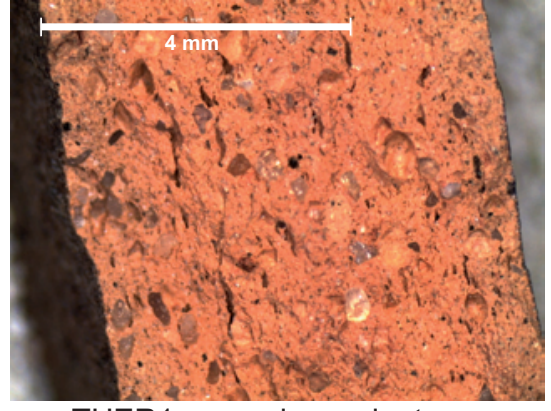

THER1 en mode oxydant

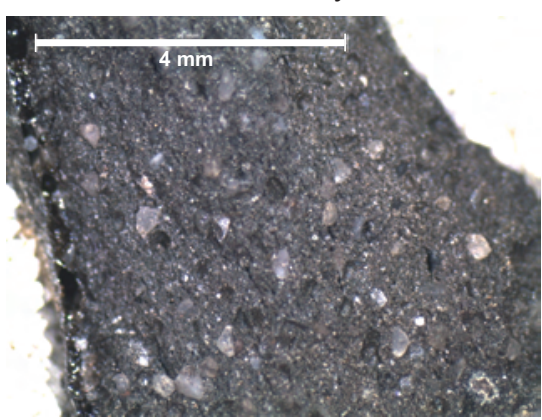

THER1 en mode réducteur

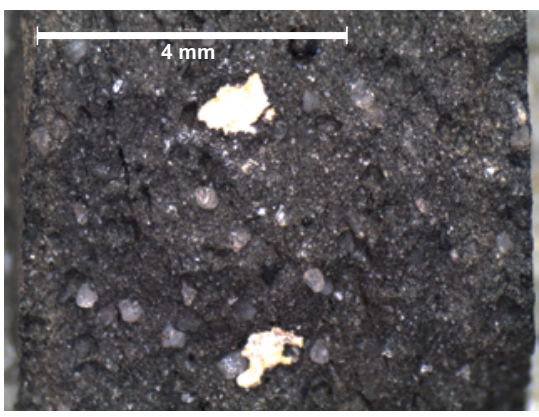

THER2

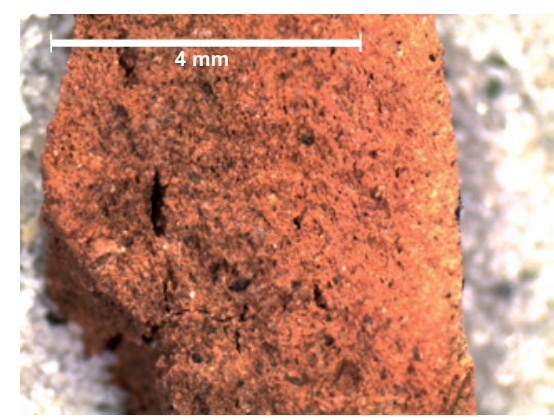

THER3 en mode oxydant

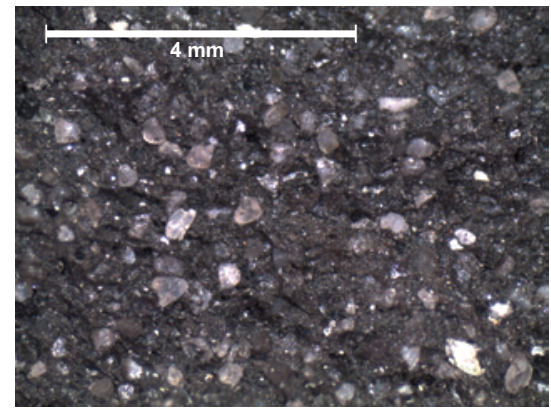

THER4 par la présence de pores longitudinaux, de petite à moyenne taille, en quantité modérée.

Le second groupe (THER2) ne caractérise que des éléments en pâte grise. Il est absent de l'horizon de l'habitat et se rencontre uniquement au sein de l'ensemble céramique issu du four (NMI 5, soit un peu moins de $5 \%$ du corpus), ainsi que pour un fragment de brique issu de son comblement. Il se définit par un cœur gris très homogène et des bords extérieurs présentant une tonalité plus foncée et un toucher lisse. La trame générale est beaucoup plus fine que le premier groupe. La pâte est extrêmement cuite, quasi vitrifiée, ce qui lui confère un aspect assez brillant. Il existe deux niveaux de quartz bien distincts: un premier constitué de grains de taille moyenne, subarrondis et transparents en quantité moyenne et un second de grains très petits, arrondis à subarrondis, en très grande quantité. À ceux-ci s'ajoutent de rares nodules noir brillant subarrondis de taille moyenne, et quelques concrétions blanches non identifiées, de forme irrégulière, mais d'assez grand module.

Seuls deux individus provenant du comblement du four peuvent être rattachés au groupe THER3. Celuici se rencontre également (NMI 2) en contexte d'habitat plus tardif. Il caractérise, tout comme le groupe THER1, aussi bien des éléments cuits en atmosphère oxydante que réductrice. La pâte est lisse, de couleur homogène, à cœur comme en surface, du gris à l'orange selon le mode de cuisson. Sa structure est extrêmement fine, de même que la granulométrie. Les inclusions se composent de nombreux grains de quartz subarrondis translucides, des nodules noirs brillants subarrondis présents en quantité moyenne et d'occasionnels pores longitudinaux. On notera également la présence de rares oxydes de fer rouges, anguleux, généralement de grande taille. Ce groupe de pâtes n'est pas sans rappeler celui des pâtes scaldiennes, que l'on rencontre par exemple à Lille ou Tournai. Des études chimiques permettraient de confirmer cet approvisionnement exogène. L'éventail morphologique du groupe est varié, avec une tèle en TCRgl et un pot à provision en TCG en contexte d'habitat; un pichet (PI2) en pâte grise et un poêlon en TCRgl (PO1) pour le mobilier issu du comblement du four.

Les échantillons de pâte prélevés sur quarante-neuf boudins, un carreau et deux briques recueillis dans le comblement du four appartiennent au groupe THER4. Il faut noter que celui-ci caractérise uniquement des éléments modelés et non tournés, à la différence des

FIG. 12. - Groupes de pâtes du mobilier issu du comblement de la chambre de chauffe.

V. Vincent. 


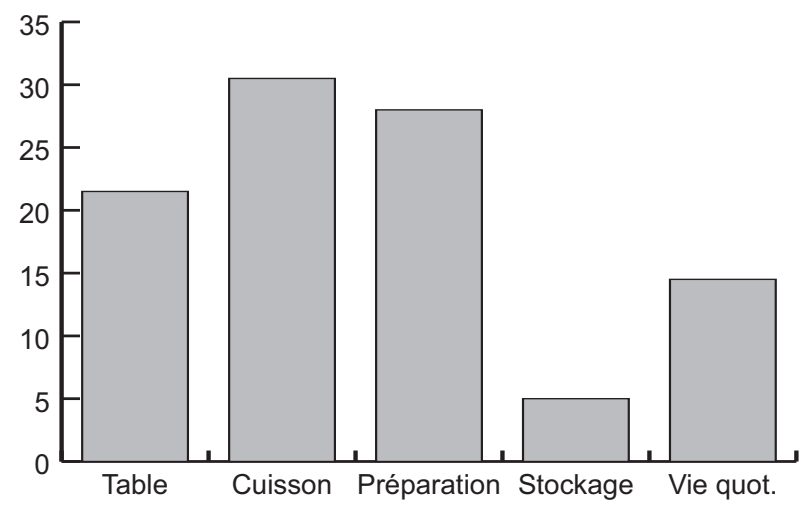

FIG. 13. - Éventail morphologique du mobilier issu du remplissage de la chambre de chauffe (répartition en pourcentage des NMI).

trois groupes précédents. Cela joue bien évidemment sur l'organisation des inclusions. Ce groupe est très proche de THER1, dans sa structure et ses composants. La principale différence est le nombre de grains de quartz de taille moyenne, qui sont présents ici en très grande quantité. La structure secondaire est, quant à elle, parfaitement similaire, composée de très nombreux grains de quartz de très petite taille pris dans une matrice très fine. Sans doute faut-il considérer THER4 comme une variante de THER1, moins dégraissée et réservée aux éléments d'architectures. Seules des analyses chimiques pourraient confirmer un approvisionnement aux mêmes gisements pour les groupes THER1, THER2 et THER4.

\subsection{Aspects morphologiques}

Le corpus céramique issu du comblement du four est morphologiquement très diversifié, avec trois grandes catégories : la vaisselle de table $(21,6 \%)$, de cuisson $(30,4 \%)$ et de préparation $(28,4 \%)$. Les pots pour le stockage $(4,9 \%)$ et des objets de la vie quotidienne $(14,7 \%)$ sont également présents, mais dans de moindres proportions (fig. 13, tab. 2).

\subsubsection{La vaisselle de table: écuelles, pichets et gobelets}

La vaisselle de table (fig. 14) regroupe trois types de contenants: l'écuelle pour la consommation de bouillie ou de soupe, le pichet et le gobelet, tous deux associés à la boisson. Les assiettes sont absentes du corpus. D'un point de vue technique, la vaisselle de table est presque exclusivement en TCG et, généralement, en pâte THER1, avec seulement un pichet en
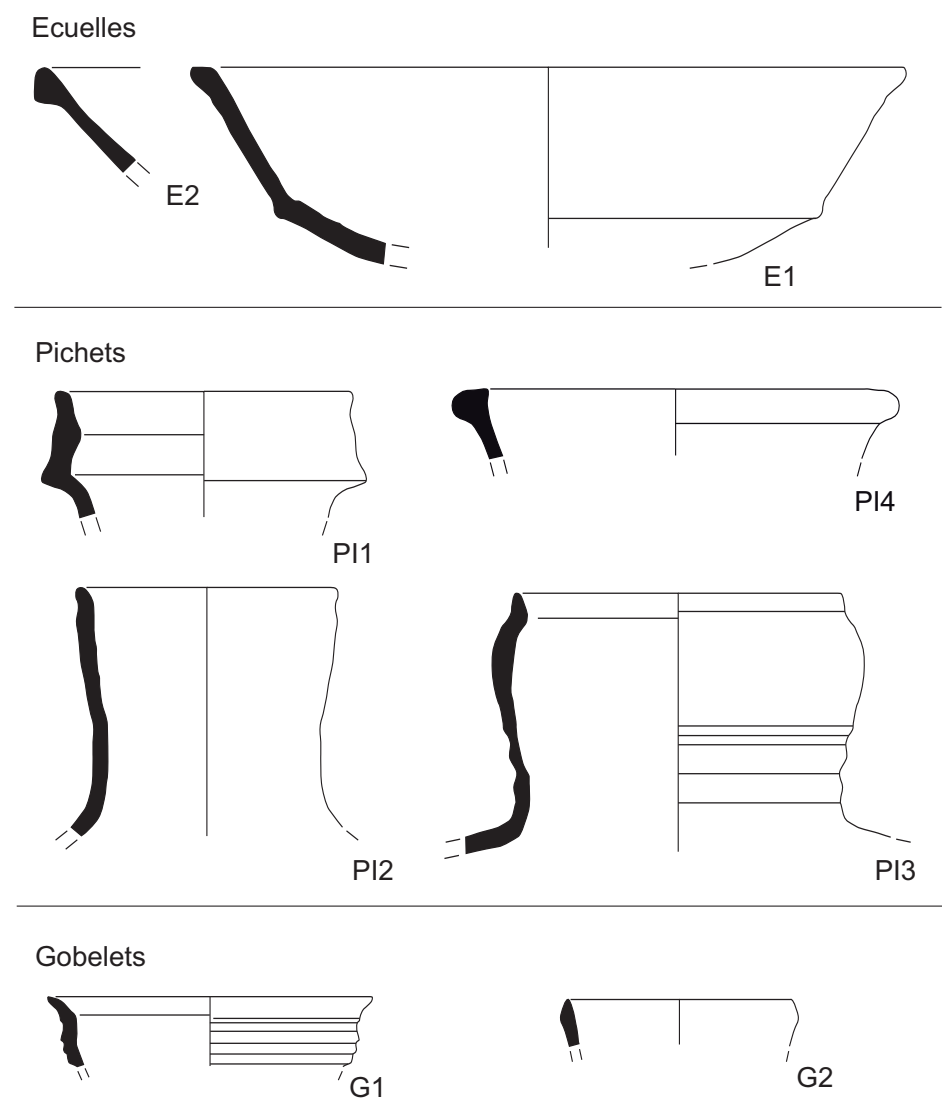

FIG. 14. - Typologie du mobilier céramique issu du comblement de la chambre de chauffe : la vaisselle de table. DAO : V. Vincent et S. Quévreux. 


\begin{tabular}{|c|c|c|c|c|c|c|}
\hline & NMI & Typologie & Description & Technique & Fabrique & NMI \\
\hline \multicolumn{7}{|c|}{ La vaisselle de table } \\
\hline \multirow[t]{2}{*}{ Ecuelles } & \multirow[t]{2}{*}{7} & E1 & Ecuelle à lèvre confondue et panses évasée & TCG & THER1 & $\frac{5}{5}$ \\
\hline & & E2 & Ecuelle à lèvre élargie de section triangulaire & TCG & THER1 & $\overline{2}$ \\
\hline \multirow{6}{*}{ Pichets } & \multirow{6}{*}{13} & PI1 & Pichet à lèvre en bandeau légèrement saillant et col droit & TCG & THER1 & $\overline{1}$ \\
\hline & & PI2 & Pichet à col droit, légèrement évasé, et lèvre confondue à bout arrondi & TCG & $\begin{array}{ll}\text { THER1 } \\
\text { THER3 }\end{array}$ & 1 \\
\hline & & PI3 & Pichet à col cannelé et galbé avec un léger renflement interne & TCG & THER1 & $\frac{7}{7}$ \\
\hline & & PI4 & Pichet à lèvre éversée et bout élargi et arrondi & TCG & THER1 & 1 \\
\hline & & Ind & & TCG & THER1 & 1 \\
\hline & & & & TCR & THER1 & 1 \\
\hline \multirow{2}{*}{ Gobelets } & \multirow{2}{*}{2} & G1 & Gobelet à col cannelé galbé et lèvre éversée & TCG & THER1 & $\overline{1}$ \\
\hline & & $\mathrm{G} 2$ & Gobelet à lèvre confondue et extrémité affinée & TCG & THER1 & 1 \\
\hline \multicolumn{7}{|c|}{ La céramique de cuisson } \\
\hline \multirow{3}{*}{ Marmites } & \multirow{4}{*}{4} & M1 & Marmite à lèvre recourbée sur l'extérieur. de section quadrangulaire & TCG & THER1 & $\overline{1}$ \\
\hline & & & & TCG & THER2 & \\
\hline & & M2 & Marmite à lèvre éversée et bout élargi et arrondi & TCG & THER1 & 1 \\
\hline \multirow{3}{*}{ Couvercles } & & M3 & Marmite à lèvre éversée et inflexion interne & TCG & THER1 & 1 \\
\hline & \multirow[t]{2}{*}{6} & $\mathrm{C} 1$ & Couvercle à lèvre pliée vers l'extérieur, à bout arrondi & TCRgl & THER 1 & $\begin{array}{l}1 \\
2\end{array}$ \\
\hline & & $\mathrm{C} 2$ & Couvercle à lèvre éversée et confondue & TCG & THER1 & 3 \\
\hline \multirow{6}{*}{ Poêlons } & \multirow{6}{*}{21} & & & TCG & THER1 & $\overline{1}$ \\
\hline & & PO1 & Poêlon à lèvre élargie et arrondie sur son parement externe & TCRgl & THER1 & 3 \\
\hline & & & & TCRgl & THER3 & 1 \\
\hline & & & & TCG & THER1 & 7 \\
\hline & & $\mathrm{PO} 2$ & Poêlon à lèvre recourbée en crochet sur l'extérieur & TCR & THER1 & 3 \\
\hline & & & & TCRgl & THER1 & 6 \\
\hline \multicolumn{7}{|l|}{ La préparation } \\
\hline \multirow{3}{*}{ Terrines } & \multirow{3}{*}{4} & TR1 & Terrine à lèvre éversée et élargie, panse galbée & TCG & THER1 & $\frac{2}{2}$ \\
\hline & & TR2 & Terrine à lèvre éversée et repliée sur l'extérieur & TCG & THER1 & 1 \\
\hline & & TR3 & $\begin{array}{l}\text { Terrine à lèvre éversée à bout arrondi rentrant, formant une petite } \\
\text { inflexion interne }\end{array}$ & TCG & THER & 11 \\
\hline \multirow{5}{*}{ Tèles } & \multirow{5}{*}{25} & & & TCG & THER1 & 20 \\
\hline & & T1 & Tèle à bandeau peu saillant & TCG & THER2 & 2 \\
\hline & & & & TCGgl & THER1 & 1 \\
\hline & & $\mathrm{T} 2$ & Tèle à bandeau confondu, avec une légère saillance & TCG & THER1 & $\frac{1}{1}$ \\
\hline & & 12 & & TCG & THER2 & 1 \\
\hline \multicolumn{7}{|l|}{ Le stockage } \\
\hline \multirow{2}{*}{ Pots à provisions } & \multirow{2}{*}{5} & PAP1 & Pot à provision à lèvre droite, élargie et bout plat & TCG & THER1 & $\frac{2}{2}$ \\
\hline & & PAP2 & Pot à provision à lèvre repliée vers l'extérieur, de section quadrangulaire & TCG & THER1 & 3 \\
\hline \multicolumn{7}{|c|}{ Les éléments de la vie quotidienne } \\
\hline & & CF1 & $\begin{array}{l}\text { Couvre-feu à lèvre élargie de section quadrangulaire, liaison col/panse } \\
\text { marquée par une légère excroissance de la pâte }\end{array}$ & TCG & $\begin{array}{l}\text { THER1 } \\
\text { THER2 }\end{array}$ & $\begin{array}{l}2 \\
1\end{array}$ \\
\hline Couvre-feux & 10 & $\mathrm{CF} 2$ & $\begin{array}{l}\text { Couvre-feu à col irrégulier, lèvre éversée avec un parement interne } \\
\text { concave, liaison col/panse marquée par un décor digité }\end{array}$ & TCG & THER1 & $\frac{1}{4}$ \\
\hline & & CF3 & $\begin{array}{l}\text { Couvre-feu à lèvre légèrement élargie et extrémité plate, liaison } \\
\text { lèvre/col marquée d'un sillon en relief }\end{array}$ & TCG & THER1 & 3 \\
\hline Pots de fleurs & 5 & PF1 & Pot de fleur à lèvre éversée à inflexion légèrement arrondie & TCG & THER1 & 2 \\
\hline Pots de neurs & J 3 & PF2 & Pot de fleur à lèvre rabattue sur l'extérieur & TCR & THER1 & 3 \\
\hline Indéterminé & 1 & & & TCG & THER1 & $\frac{1}{1}$ \\
\hline Total & 103 & & & & & 103 \\
\hline
\end{tabular}

Abréviations utilisées : TCG = Terre cuite grise $/ \mathrm{TCGgl}=$ Terre cuite grise glaçurée $/ \mathrm{TCR}=$ Terre cuite rouge $/ \mathrm{TCRgl}=$ Terre cuite rouge glaçurée.

Tableau 2. - Comptage en NMI du mobilier issu du comblement de la chambre de chauffe par catégorie typologique, catégorie technique et groupes de pâte. 

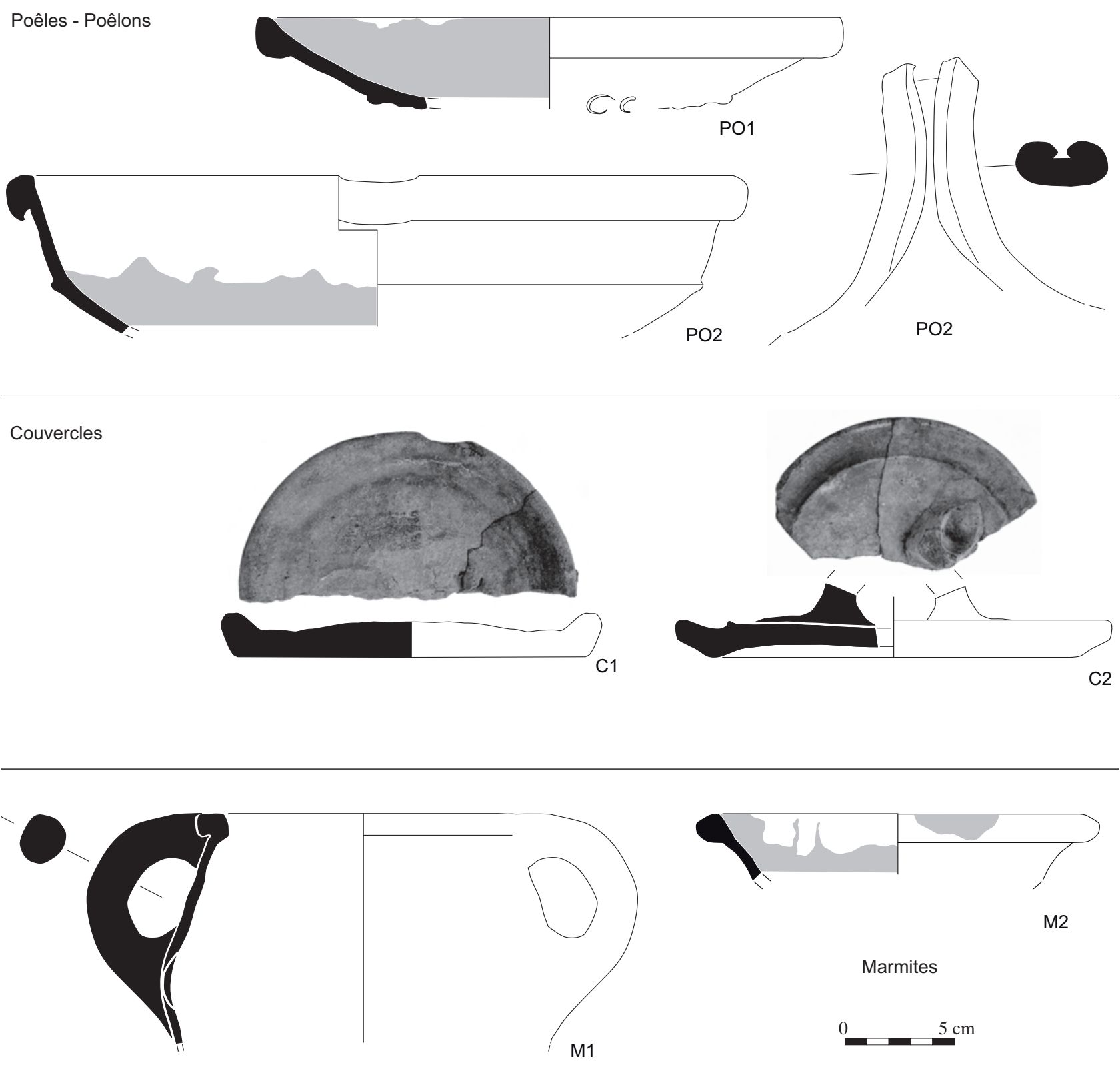

FIG. 15. - Typologie du mobilier céramique issu du comblement de la chambre de chauffe : la vaisselle de cuisson. DAO : V. Vincent.

THER3. Le fragment de pichet en grès importé, dont la pâte rappelle les productions de l'atelier de Siegburg, doit également être rattaché à cet ensemble.

Les écuelles se partagent entre deux types: E1 à lèvre confondue, avec cinq individus, et E2 à lèvre élargie de section triangulaire, avec deux individus. Quatre types de pichets ont pu être identifiés, mais seul le type PI3, à col cannelé très galbé et lèvre à renflement intérieur, semble véritablement représentatif de la production locale avec sept individus, contre un à deux pour les type PI1, PI2 et PI4. Enfin, deux variantes de gobelets ont été recensés: le type G1, à col cannelé et lèvre éversée et le type G2 à lèvre confondue légèrement amincie.

\subsubsection{La céramique de cuisson: la prédominance du poêlon}

Les récipients utilisés pour la cuisson des aliments (fig. 15) se répartissent en deux groupes: les marmites et couvercles qui servent à la cuisson « en mijotée » et les poêlons, utilisés pour une cuisson ouverte et frite. Le premier groupe est peu représenté avec seulement 
quatre marmites pour six couvercles. On rencontre trois types de marmites, en TCG ou en TCR: M1, à lèvre rabattue sur l'extérieur de section quadrangulaire, M2 à lèvre éversée, bombée sur l'extérieur et $\mathrm{M} 3$, à lèvre éversée et inflexion interne, probablement d'origine intrusive. Les couvercles peuvent être en TCG ou en TCRgl. Trois fragments présentent une lèvre repliée en partie supérieure (type $\mathrm{C} 1$ ), trois autres une lèvre éversée (type C2).

Les poêlons se partagent uniformément entre TCG et TCRgl. La glaçure est le plus souvent coulée à l'intérieur du récipient et généralement de couleur orange. Le premier type, PO1 (NMI 5), est de faible profondeur, avec une lèvre élargie et arrondie. Le second, PO2, domine le corpus avec seize individus. La lèvre est ici recourbée en crochet. L'élément de préhension est formé d'une plaque de pâte, dont les deux extrémités sont rabattues vers l'intérieur.

D'un point de vue technique, les récipients appartiennent généralement à la fabrique THER1. Notons toutefois une marmite de type M1 en THER2 et un poêlon PO1 en THER3.

\subsubsection{La préparation: terrines et tèles}

Les ustensiles de préparation (fig. 16) se répartissent entre les terrines (NMI 4) et les tèles, beaucoup plus nombreuses avec vingt-cinq individus. Les formes de terrines sont diversifiées, avec trois types pour quatre individus: le type TR1, à lèvre éversée et élargie; le type TR2, à lèvre éversée, rabattue sur l'extérieur; le type TR3 à lèvre éversée et extrémité arrondi. Pour les tèles, le type T1, à lèvre en bandeau peu saillant, est tout à fait représentatif de la production locale (NMI 23). Le type T2, avec seulement deux fragments est plus anecdotique. Cette catégorie de céramique est exclusivement cuite en mode réducteur. Seul un élément présente des traces de glaçure (cf. supra, 3.1). La fabrique THER1 est encore une fois largement dominante, mais trois tèles sont en THER2.

\subsubsection{Le stockage}

La céramique de stockage est ici peu représentée. Sur les cinq pots à provisions recensés (fig. 16), deux ont pu être rattachés au type PAP1 à lèvre droite, élargie et bout plat; et trois au type PAP2 à lèvre repliée

\footnotetext{
6. - RoY, BARBÉ 1998, p. 90, fig. 58 (2e moitié XV $\mathrm{XV}^{\mathrm{e}} 1^{\mathrm{er}}$ quart XVI $\mathrm{X}^{\mathrm{e}}$ siècle - pâte différente); Belot, CANut 1997, p. 130, fig. 28 (fin XVe-début $\mathrm{XVI}^{\mathrm{e}}$ siècle); BonVARLET 1998, p. 39, n 2235-19 (fin XIV ${ }^{\mathrm{e}}$-début $\mathrm{XVI}^{\mathrm{e}}$ siècle comme tèle); VERHAEGHE 1987, p. 220, fig. $12\left(\mathrm{XIV}^{\mathrm{e}}\right.$ siècle, comme tèle et avec une pâte différente).

7. - Roy 1997b, fig. 12; BARBÉ et al. 1997a, fig. 22-23; BARBÉ et al. 1997b, fig. 3 ; BARRET et al. 1996, p. 48, fig. 15.

8. - Alvey, Verhaeghe 1981, fig. 26 (XIII ${ }^{\mathrm{e}}$-milieu XIV ${ }^{\mathrm{e}}$ siècle); Barbé
}

vers l'extérieur, de section quadrangulaire. Tous ces éléments sont en TCG et en THER1.

\subsubsection{Les éléments de la vie quotidienne}

À travers le terme « vie quotidienne», sont regroupés les couvre-feux et pots de fleurs, habituellement classé en catégorie « divers ». Dans l'ensemble étudié, on dénombre dix couvre-feux et cinq pots de fleurs (fig. 16). Trois types de couvre-feux ont été différenciés: CF1, à lèvre élargie de section quadrangulaire légèrement arrondie (NMI 3); CF2 à col irrégulier, lèvre éversée avec un parement interne concave et une liaison col/panse marquée par un décor digité (NMI 4); CF3 à lèvre légèrement élargie et extrémité plate (NMI 3). Ces couvre-feux sont en TCG et en THER1, à l'exception d'un individu en THER2.

Deux types de pots de fleurs ont également été identifiés: $P F 1$ à lèvre éversée à inflexion légèrement arrondie (deux fragments en TCG) et PF2 à lèvre rabattue sur l'extérieur (trois fragments en TCR), tous ces individus appartiennent à la fabrique THER1.

\section{DISCUSSION CHRONOLOGIQUE}

D'un point de vue technique (fig. 11), la terre cuite grise domine très largement le corpus étudié $(80 \%$ de TCG en NMI, $89 \%$ en NR et $1 \%$ de TCGgl) ce qui nous conduit à privilégier une datation de l'ensemble

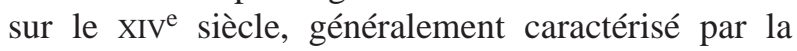
domination de la cuisson réductrice et une présence plus anecdotique de terre cuite rouge glaçurée. La présence d'un fragment de grès nous amène cependant à considérer le développement des importations des $\mathrm{XV}^{\mathrm{e}}-\mathrm{XVI}^{\mathrm{e}}$ siècles et donc à privilégier la fin du $\mathrm{XIV}^{\mathrm{e}}$ siècle ou le début du $\mathrm{XV}^{\mathrm{e}}$ siècle.

L'ensemble des références morphologiques renvoie au même balancement chronologique. L'écuelle E2 trouve ainsi des comparaisons à Douai et en Flandre dans des contextes du XIv e siècle et à Boulogne-surMer et Saint-Omer pour la fin du XV $\mathrm{XV}^{\mathrm{e}}$ siècle et le début $\mathrm{XVI}^{\mathrm{e}}$ siècle ${ }^{6}$. De même, l'écuelle E1 peut être comparée à des éléments de la seconde moitié $\mathrm{du} \mathrm{XV}^{\mathrm{e}}$-début $\mathrm{XVI}^{\mathrm{e}}$ siècle à Saint-Omer ${ }^{7}$. Les pichets PI3 et PI4 sont plutôt caractéristiques de périodes plus anciennes. On rencontre généralement le premier en contexte des XIII $-\mathrm{XIV}^{\mathrm{e}}$ siècle en Flandre, à Saint-Omer, Lens, Lille et Courtrai ${ }^{8}$, le second en contexte du XIII ${ }^{\text {e }}$ siècle $^{9}$.

\footnotetext{
et al. 1997a (milieu XIII ${ }^{\mathrm{e}}$ siècle), fig. 31; BLIECK 1996 (milieu XIII $^{\mathrm{e}}$ $1^{\text {er }}$ quart XIV ${ }^{\mathrm{e}}$ siècle - type 4 - et XIV ${ }^{\mathrm{e}}$ siècle); DESPRIET 1998, type 5 (1250-1325); De GRoOTE 2008, L130b (XIII'-fin XIV ${ }^{\mathrm{e}}$ siècle); MARCY 2004, n 5906.91 (XIV $^{\mathrm{e}}$ siècle); RoY 1997a, p. 132, fig. 6 (2e tiers XIII ${ }^{\mathrm{e}}$ siècle).

9. - De Groote 2008, L63 ou L68 (1150-1300); VinCENT 2010, pl. 2, $\mathrm{n}^{\circ} 28$; DEBS, MARCY 2006, p. 34 ; MARCY 2004, n 5911.107.
} 
Terrines

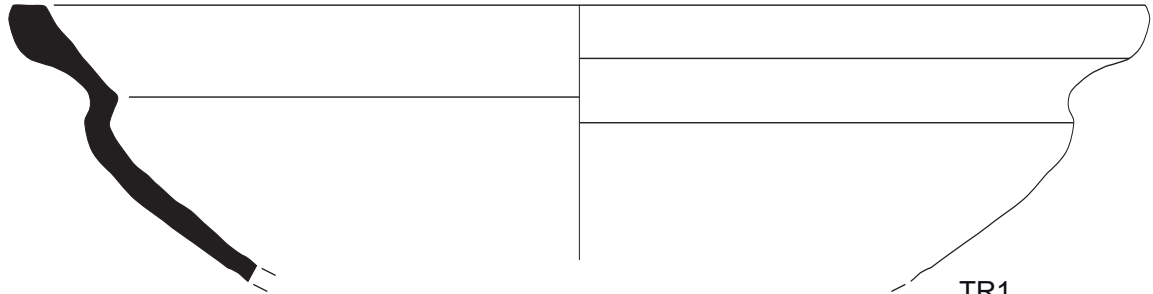

TR1

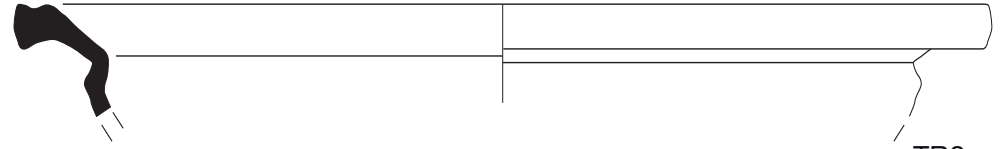

TR2

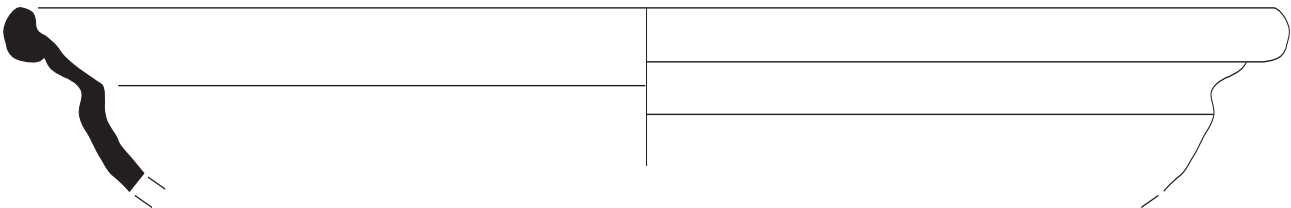

TR3

Tèles
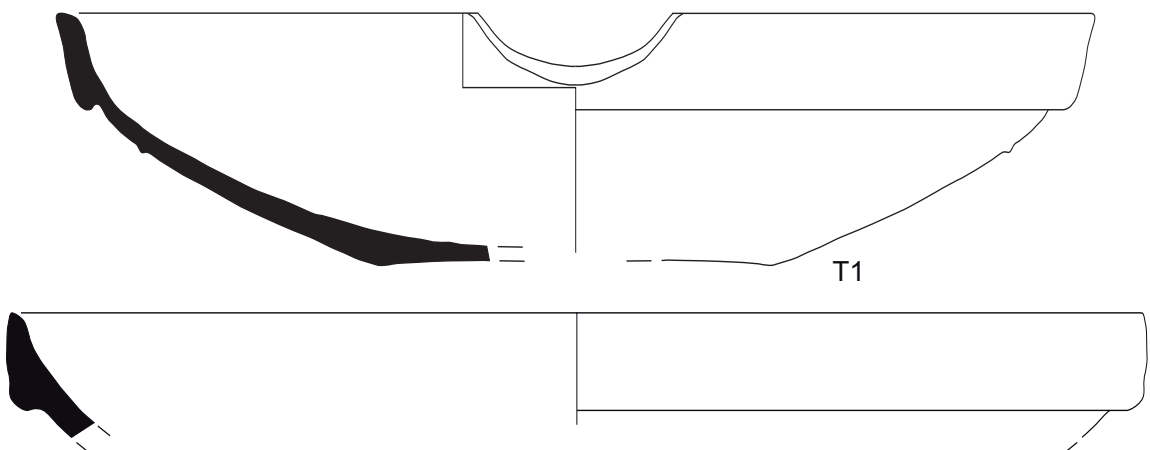

$\mathrm{T} 2$

Couvre-feux

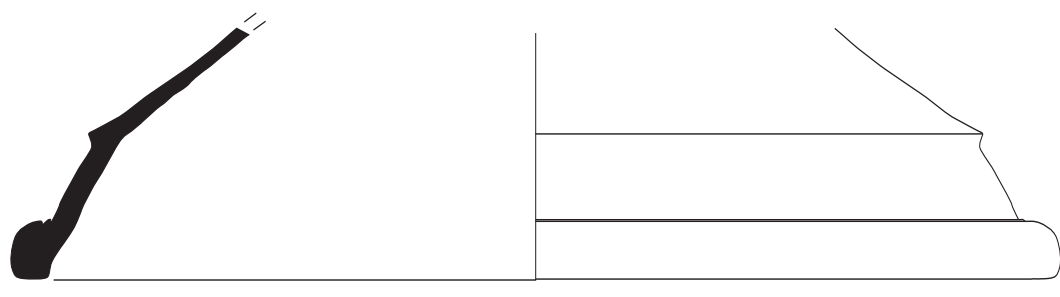

CF1
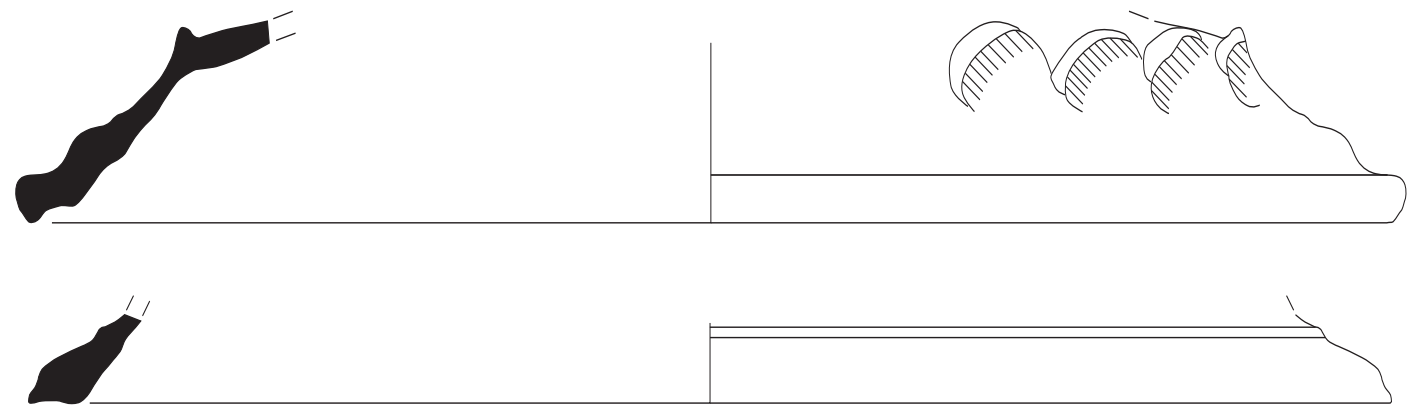

$\mathrm{CF}^{3}$

Pots à provision
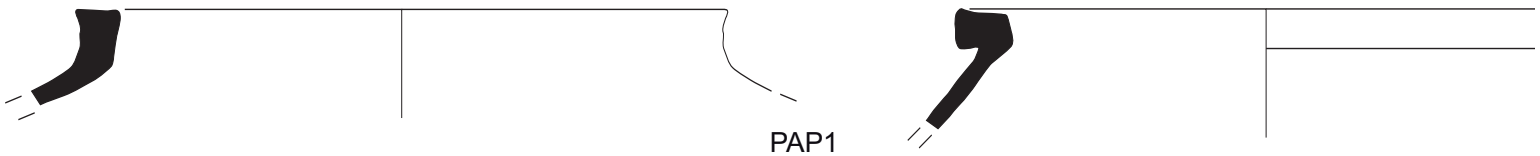

FIG. 16. - Typologie du mobilier céramique issu du comblement de la chambre de chauffe : la vaisselle de préparation, de stockage et les objets de la vie quotidienne. DAO V. Vincent. 
Le pichet PI2 est un peu plus tardif (XIv ${ }^{\mathrm{e}}$-début $\mathrm{XVI}^{\mathrm{e}}$ siècle) à Ardres, Saint-Omer ou Lille ${ }^{10}$. Le gobelet $\mathrm{G} 2$ trouve lui aussi un homologue en contexte lillois du XIV siècle ${ }^{11}$. PO1 rappelle les poêlons trouvés à Lille, Ardres et Saint-Omer dans la seconde moitié du XIV ${ }^{\mathrm{e}}$ siècle ou le début du $\mathrm{XV}^{\mathrm{e}}$ siècle $^{12}$. En revanche, le poêlon à crochet $\mathrm{PO} 2$ rappelle un exemplaire audomarois de la fin du $\mathrm{XV}^{\mathrm{e}}$-début $\mathrm{XVI}^{\mathrm{e}}$ siècle ${ }^{13}$. La marmite M1 appartient au même intervalle chronologique que PO1 en Flandre ou en Wallonie ${ }^{14}$, et la marmite $\mathrm{M} 2$ au même intervalle que $\mathrm{PO} 2$, à Lille, en Flandre ou à Saint-Omer. Les terrines sont sur ce même entre-deux des XIV et $\mathrm{XV}^{\mathrm{e}}$ siècles en contextes ardrésien, audomarois, et flamands ${ }^{15}$. La tèle $\mathrm{T} 1$ à bandeau peu saillant est quant à elle véritablement caractéristique des contextes de la fin du XIV ${ }^{\mathrm{e}}$ siècle et du début $X V^{\mathrm{e}}$ siècle, à Douai notamment ${ }^{16}$. Elle préfigure la tèle à bandeau saillant des $\mathrm{XV}^{\mathrm{e}}$ et $\mathrm{XVI}^{\mathrm{e}}$ siècles. Les pots de stockage PAP2 trouvent des homologues flamands et audomarois de la fin du $\mathrm{XIV}^{\mathrm{e}}$ au $\mathrm{XV}^{\mathrm{e}}$ siècle ${ }^{17}$; PAP1 rappelle des éléments de Saint-Omer des $\mathrm{XV}^{\mathrm{e}}$-début $\mathrm{XVI}^{\mathrm{e}}$ siècle ${ }^{18}$. Les variations morphologiques des objets de la vie quotidienne comme les couvre-feux et les pots de fleur sont moins marquées que pour les autres catégories. Le couvre-feu CF1 trouve des comparaisons en contextes flamands du $\mathrm{XIII}^{\mathrm{e}}$ au $\mathrm{XV}^{\mathrm{e}}$ siècle $^{19}$; CF3 rappelle les productions d'Ardres de la seconde moitié du XIV siècle ${ }^{20}$. D'une façon générale, de nombreuses formes rappellent les productions de l'atelier d'Ardres, daté de la seconde moitié du XIV siècle, mais les formes des poêlons, des terrines et des écuelles nous incitent à prendre en compte le $\mathrm{XV}^{\mathrm{e}}$ siècle. D'où la proposition d'une datation du mobilier céramique issu du comblement sur un horizon de la fin du XIV ${ }^{\mathrm{e}}$ ou le début $\mathrm{XV}^{\mathrm{e}}$ siècle.

L'utilisation de boudin d' argile cuite pour supporter la charge est compatible avec cette datation. Cette technique est régulièrement utilisée dans les quelques ateliers régionaux datés des $\mathrm{XIV}^{\mathrm{e}}$ et $\mathrm{XV}^{\mathrm{e}}$ siècles. C'est le cas notamment à Guînes, avec deux structures de cuisson de la seconde moitié du XIV ${ }^{\text {e }}$ siècle ou du début $\mathrm{du} \mathrm{XV}^{\mathrm{e}}$ siècle mises au jour à l'occasion des

10. - Roy 1997a, p. 135, fig. 13 (milieu XV $\mathrm{XV}^{\mathrm{e}}$ siècle ou 1500-1525); Blieck 1996, p. 218, fig. 22 (XIV ${ }^{\mathrm{e}}$ siècle); PourIEL 2002, fig. 74 $\left(2^{\mathrm{e}}\right.$ moitié XIV ${ }^{\mathrm{e}}$ siècle).

11. - BLIECK 1996, p. 218, fig. 24.

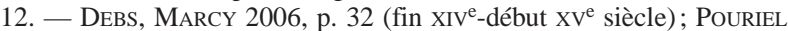
2002, fig. 10 (2 $2^{\mathrm{e}}$ moitié XIV ${ }^{\mathrm{e}}$ siècle); RoY, BARBÉ 1998, p. 90, fig. 47 (fin XIV siècle).

13. - Roy, BARBÉ 1998, p. 90, fig. 48.

14. - De Groote 2008, L146 (1375-1475); Roy 1997a, p. 99, fig. 14

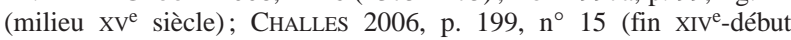
$X V^{\mathrm{e}}$ siècle).

15. — Terrine TR1 : De GROOTE 2008, L112 (1300-1400); RoY 1997a, p. 77, fig. 14 ( $2^{\mathrm{e}}$ moitié $X V^{\mathrm{e}}$ siècle); PourIEL 2002, fig. 40 (2e moitié XIV ${ }^{\mathrm{e}}$ siècle). Terrine TR2: De Groote 2008, L110 (1200-1550); RoY fouilles de la RD $244^{21}$, à $\operatorname{Ardres}^{22}$ ou sur le site de l'ancien Arsenal de Douai, avec deux fours de potiers également datés de la seconde moitié du $\mathrm{XIV}^{\mathrm{e}}$ ou du début du XV $\mathrm{Xv}^{\mathrm{e}}$ siècle $^{23}$.

Des prélèvements pour analyse archéomagnétique ont été réalisés sur le sol de la chambre de chauffe par A. Genevey (UMR CNRS 171, Centre de recherche et de restauration des musées de France) et N. Warmé (Inrap). Les directions magnétiques individuelles obtenues sont apparues fortement allongées suivant les inclinaisons et ce biais observé dans la distribution des directions n'a pas permis de définir avec confiance une direction magnétique moyenne fiable du four de Thérouanne qui permettrait de le dater (mesures réalisées au laboratoire de paléomagnétisme de l'Institut de physique du globe de Paris) ${ }^{24}$. Les directions individuelles présentent toutes une inclinaison trop faible par rapport aux inclinaisons géomagnétiques connues en France depuis 2000 ans. Il est cependant intéressant de noter que les inclinaisons les plus faibles observées depuis 2000 ans sont obtenues autour de l'an 1400 (les déclinaisons obtenues pour le four de Thérouanne étant par ailleurs compatibles avec cet âge), ce qui est un indice supplémentaire pour conforter la datation archéologique estimée à partir du mobilier céramique.

La découverte d'un four de potier intra muros montre que, malgré les risques d'incendie, « l'artisanat du feu »n'est pas systématiquement tenu à l'écart des centres urbains médiévaux. Le soin apporté à la construction de la structure de cuisson, ses dimensions, les traces de curage ou le réemploi de matériaux liés à la production céramique (boudins d'argile cuite) dans l'élévation des murets de soutènement sont des indices de l'importance et de la longévité de cette production céramique en cœur de ville. Faute d'une fouille plus extensive et exhaustive, il est cependant difficile d'appréhender l'emprise totale de l'atelier, son organisation interne et le cadre chronologique de son fonctionnement. Les barres d'argile en réemploi témoignent-elles de réfections importantes d'une

1997a, p. 77, fig. 16 ( $2^{\mathrm{e}}$ moitié $X V^{\mathrm{e}}$ siècle). Terrine TR3: BonvARLET 1998 , p. 38 (début $\mathrm{XV}^{\mathrm{e}}$ siècle).

16. - Belot, Canut 1997, p. 139, fig. 26 (fin $X V^{\mathrm{e}}$-début $X V I^{\mathrm{e}}$ siècle); BONVARLET 1998, p. 39 (fin XIV ${ }^{\mathrm{e}}$-début XV $\mathrm{XV}^{\mathrm{e}}$ siècle).

17. - VerHAEGHE 1987, p. 220, fig. 11 (comme marmite, fin XIV ${ }^{\mathrm{e}}$ début $\mathrm{XV}^{\mathrm{e}}$ siècle); BARBÉ, RoY 1994, p. 20, fig. 3 ( $\mathrm{XV}^{\mathrm{e}}$ siècle); RoY $1997 \mathrm{a}$, p. 146 , fig. 8 ( $\mathrm{XV}^{\mathrm{e}}$ siècle).

18. - Roy 1997a, p. 147, fig. 13 et BARBÉ et al. 1997a.

19. - De Groote 2008, L94A.

20. - Pouriel, 2002, type 1.

21. - WiLlot 2009.

22. - POURIEL 2002.

23. - DEMOLON 1990.

24. - Genevey et al. 2011. 
seule et même structure de production ou proviennent-elles d'autre(s) four(s), contemporain(s) ou antérieur(s), situé(s) à proximité immédiate? Le groupe de pâte THER1, sans doute caractéristique de la production locale de la fin $\mathrm{du} \mathrm{XIV}^{\mathrm{e}}$ ou du début du $\mathrm{XV}^{\mathrm{e}}$ siècle, est encore très majoritaire au sein du mobilier céramique issu des niveaux d'occupation ou de destruction de l'habitat de la fin $\mathrm{du} \mathrm{XV}^{\mathrm{e}}$ ou du début du $\mathrm{XVI}^{\mathrm{e}}$ siècle. Une activité potière s'approvisionnant aux mêmes gisements d'argile se poursuit donc sans doute, sur le site ou au pourtour de la cité, jusqu'à sa destruction par Charles Quint. Un réexamen, à la lumière de ces données nouvelles, de quelques ensembles céramiques médiévaux issus des fouilles archéologiques régionales permettrait également de mieux appréhender l'aire de diffusion et l'organisation des réseaux de distribution des productions de Thérouanne.

Mots-clés: Pas-de-Calais, Thérouanne, artisanat, céramique, Bas Moyen Âge.

\section{Bibliographie}

Alvey, Verhaeghe 1981 : Alvey R.-C., Verhaeghe F., «Medieval pottery from Wissant (N. France) », Medieval ceramics, 5, 1981, p. 35-43.

Arcelin, Tuffreau-Libre 1998 : Arcelin P., TuffreauLIBRE M. (dir), La quantification des céramiques. Conditions et protocole, Glux-en-Glenne, 1998, 157 p. (Collection Bribracte, 2)

BARbÉ, Roy 1994 : Barbé H., Roy E., Saint-Omer, rue Allent. Construction de 31 garages, 1994. (Rapport de diagnostic archéologique inédit, SRA Nord/Pas-de-Calais, Lille)

BARbÉ et al. 1997a : BARBÉ H., Deschodt L., Routier J.-C., Roy E., Le site du quai des Salines à Saint-Omer. De l'aménagement d'un réseau hydrographique à l'urbanisation industrielle d'une ville médiévale, 1997. (Rapport de fouille inédit, SRA Nord/Pas-de-Calais, Lille)

Barbé et al. 1997b: BARBÉ H., Deschodt L., Routier J.-C., RoY E., Le site des anciennes casernes d'Albret à Saint-Omer. De l'aménagement d'un réseau hydrographique à l'urbanisation d'une ville médiévale. "Les abords de Vinquai », 1997. (Rapport de fouille inédit, SRA Nord/Pas-de-Calais, Lille)

Barret et al. 1996 : Barret M., Roy E., VANhille G., SaintOmer «Esplanade » (Pas-de-Calais), 1996. (Rapport de diagnostic archéologique inédit, SRA Nord/Pas-de-Calais, Lille) Belot, Canut 1997 : Belot E., Canut V., Le cru et le cuit. Dieux oubliés et pots cassés du Boulonnais. Les dieux de Boulogne durant l'Antiquité gallo-romaine. Vestiges de l'équipement céramique de cuisine et de table du Moyen Âge à l'époque moderne, Boulogne-sur-Mer, 1997. (Éléments d'archéologie boulonnaise, 5)

BERNARD 1980 : BERNARD H., « Remarques et hypothèse sur le développement urbain de Thérouanne (Pas-de-Calais) », Septentrion, 10, 1980, p. 41-60.
Blamangin 2011 : Blamangin O. (dir.), Thérouanne, rue Saint Jean: atelier de potier médiéval au cour de la ville fortifiée, 2011. (Rapport de diagnostic archéologique inédit, SRA Nord/Pas-de-Calais, Lille)

Blamangin et al. 2012 : Blamangin O., Devred V., LERICHE B., « L'apport de l'archéologie préventive à la connaissance de la topographie antique de la ville de Thérouanne », Revue du Nord, ce vol.

BlieCK 1996 : BlieCK G., «Céramiques et objets divers du XIV $^{\mathrm{e}}$ siècle mis au jour dans une latrine du château dit de Courtrai à Lille », dans Piton D. (éd.), La céramique très décorée dans l'Europe du nord-ouest ( $X^{e}-X V^{e}$ siècles), Actes du colloque de Douai du 7-8 avril 1995, Berck-sur-Mer, p. 183-232. (Nord-Ouest Archéologie, 7)

Bonvarlet 1998 : Bonvarlet A., Un habitat médiéval rue Mongat à Douai (XIII -XVe siècle), Douai, 1998. (Archaeologia Duacensis, 12)

Challe 2006 : Challe S., « La "Cense du donjon" à Naast (Soignies). Étude du matériel céramique, fouille 2004 », Annales du cercle royal d'histoire et d'archéologie du Canton de Soignies, 38, 2006, p. 196-211.

Challe 2007-2008: Challe S., «Céramique médiévale aux Douze Césars à Tournai », Vie archéologique, 66-67, 20072008, p. 87-114.

Debs, Marcy 2006 : Debs L., Marcy T., « L'étude céramique », dans Lille, Rue des Tanneurs, vol. 2, 2006. (Rapport de fouilles archéologiques inédit, SRA Nord/Pas-de-Calais, Lille)

De Groote 2008 : De Groote K., Middeleeuws aardewerk in Vlanderen. Techniek, typologie, chronologie en evolutie van het gebuiksgoed in de region Oudenaarde in de volle en late middeleeewen (10de-16de eauw). Deel I, Bruxelles, 2008.

Delmaire 1976 : Delmaire R., Étude archéologique de la Cité des Morins (Civitas Morinorum), Arras, 1976, $410 \mathrm{p}$. (Mémoire de la commission départementale des Monuments historiques du Pas-de-Calais, 16)

Delmaire 1984 : Delmaire R., « Notes sur l'évolution urbaine de Thérouanne », dans Les villes de la Gaule Belgique au Haut Empire, Actes du colloque tenu à Saint-Riquier les 2223 et 24 octobre 1982, Revue archéologique de Picardie, (3-4), 1984, p. 223-238.

Delmaire 1994 : Delmaire R. (éd), Le Pas-de-Calais, Paris, 1994, t. 1, p. 83-98. (Carte archéologique de la Gaule, 62)

Demolon 1990 : Demolon P. (dir), Louis E., Debs L., Marlière P., Douai, l'Arsenal, Douai, 1990. (Rapport de fouille de sauvetage programmé inédit, Service archéologique du Musée de Douai)

DESPRIET 1998 : DeSPRIET P., Kortrijkse aardewerk, 12501325, Courtrai, 1998. (Archeologische en Historische Monografieën van Zuid-West Vlaanderen, 39)

Genevey et al. 2011 : Genevey A., Warmé N., Gallet Y., Le GofF M., «Rapport d'analyses archéomagnétiques effectuées au laboratoire de Paléomagnétisme de l'Institut de Physique du Globe de Paris: Étude d'un four de potier mis au jour sur le site de Thérouanne (Pas de Calais) », dans Blamangin 2011, 8 p. Gubellini 2002 : Gubellini L., "Céramique et verrerie en milieu hospitalier au XVI ${ }^{\mathrm{e}}$ siècle: l'hospice Gantois à Lille », Revue du Nord, 84 (348), 2002, p. 145-169.

Marcy 2004 : MARCY T., «Étude de la céramique Lilloise issue de l'Îlot des Tanneurs ( $3^{\mathrm{e}}$ phase de fouille: 2002$)$ ), dans Cercy C., Lille (59) Rue des Tanneurs - Les Tanneurs 3, mai 2002-avril 2003, 3, 2006. (Rapport de fouilles archéologiques inédit, SRA Nord/Pas-de-Calais, Lille) 
Méthodes D'ANALYSE de LA TERRE CUITE 1999 : « Méthodes d'analyse de la terre cuite », dans Actes de la journée d'Archéologie en Province de Liège, Ocquier, 1998, Liège, $1999,89 \mathrm{p}$.

Pouriel 2002 : Pouriel R., Le site de la maison de retraite à Ardres: étude de la céramique provenant d'une fosse dépotoir d'un atelier de potier, 2002. (Mémoire de maîtrise de l'Université de Charles-de-Gaule-Lille 3, sous la direction d'A. Muller)

Rider, TOCK 2010 : Rider J., Tock B.-M. (éd.), Le diocèse de Thérouanne au Moyen Âge. Actes de la journée d'études tenue à Lille le 3 mai 2007, Arras, 2010. (Mémoires de la Commission départementale d'histoire et d'archéologie du Pas-de-Calais, 39)

Roy 1997a: Roy E., Études typologiques de la céramique du $X_{I I I}^{e}$ au XVIII siècle dans le nord de l'Artois. Mobilier issu d'ensembles clos provenant des fouilles de Saint-Omer et Béthune, 1997. (Mémoire de maîtrise soutenu à l'EHESS, Paris).

Roy 1997b: Roy E., Calais, numéros 4, 6, 8 rue Berthois, 1997. (Rapport de diagnostic-fouille archéologique inédit, SRA Nord/Pas-de-Calais, Lille)

Roy, BARBÉ 1998 : Roy E., BARbÉ H. (avec la coll. de), « Évolution typologique et rôle domestique des céramiques de Saint-
Omer du XIII" au XVIII ${ }^{\mathrm{e}}$ siècle », Revue du Nord, 80 (328), 1998, p. 69-97.

VERHAEghe 1987 : VerhaEghe F., « La céramique en Flandre $\left(\mathrm{XIII}{ }^{\mathrm{e}}-\mathrm{XV}^{\mathrm{e}}\right.$ siècle): quelques aspects de l'évolution et de la concurrence », dans Chapelot J., Galinié H., Pilet-Lemière J. (dir), La céramique ( $V^{e}-X I X^{e}$ siècle): fabrication, commercialisation, utilisation, Actes du Congrès international d'archéologie médiévale (Paris, 4-6 octobre 1985), 1987, p. 203-225.

VinCENT 2010 : VinCENT V., «Étude céramique », dans Henton A. (dir), Trith-Saint-Léger, Nord, " îlot des Fontaines ». Traces d'occupation médiévale et approche morphologique en bordure de l'Escaut, 2010, p. 11-19. (Rapport final d'opération de diagnostic archéologique inédit, SRA Nord/Pas-de-Calais, Lille)

VINCENT (à paraître) : VINCENT V., « Étude céramique médiévale en contexte défensif: la fouille de la rue du palais Rihour à Lille », dans CERCY C. (dir), Lille, rue du Palais Rihour 2008, à paraître. (Rapport de fouille archéologique inédit)

Willot 2009 : Willot J.-M. (dir.), François S., Gapenne A., Leroy S., Masse A., Mayer A., Pineau A., Guînes (Pas-deCalais), RD244, 2009. (Rapport final d'opération de fouille inédit, Conseil général du Pas-de-Calais) 